\title{
Elastoplastic consolidation at finite strain Part 2: Finite element implementation and numerical examples
}

\author{
Ronaldo I. Borja ${ }^{a} *$, Claudio Tamagnini ${ }^{a}$, Enrique Alarcón ${ }^{\mathrm{b}}$ \\ "Department of Civil and Environmental Engineering, Stanford University, Stanford, CA 94305, USA \\ ${ }^{\mathrm{h}}$ Departamento de Mecánica Estructural y Construcciones Industriales, Universidad Politécnica de Madrid, 28006 Madrid, Spain
}

\begin{abstract}
A mathematical model for finite strain elastoplastic consolidation of fully saturated soil media is implemented into a finite element program. The algorithmic treatment of finite strain elastoplasticity for the solid phase is based on multiplicative decomposition and is coupled with the algorithm for fluid flow via the Kirchhoff pore water pressure. A two-field mixed finite element formulation is employed in which the nodal solid displacements and the nodal pore water pressures are coupled via the linear momentum and mass balance equations. The constitutive model for the solid phase is represented by modified Cam-Clay theory formulated in the Kirchhoff principal stress space, and return mapping is carried out in the strain space defined by the invariants of the elastic logarithmic principal stretches. The constitutive model for fluid flow is represented by a generalized Darcy's law formulated with respect to the current configuration. The finite element model is fully amenable to exact linearization. Numerical examples with and without finite deformation effects are presented to demonstrate the impact of geometric nonlinearity on the predicted responses. The paper concludes with an assessment of the performance of the finite element consolidation model with respect to accuracy and numerical stability.
\end{abstract}

In memory of a dear friend, Bob Schiffman, for his invaluable contributions to the development of nonlinear consolidation theory.

\section{Introduction}

Compressible clays typically develop large deformations over a finite period of time. In many cases, large ground movement that results from time-dependent deformation impacts the performance of critical geotechnical structures. Time-dependent movement in clays may be attributed to the following factors [1]: (a) hydrodynamic lag, or consolidation, a transient phenomenon in which pore fluids are expelled from the soil mass; and (b) soil creep, a phenomenon which involves irreversible deformation arising from the viscous character of soil behavior. Creep deformations are rheological in nature and represent a time-dependent constitutive response, while consolidation involves a transient interaction between the solid and fluid phases and results in delayed deformation due to stress changes in the soil matrix. This paper focuses on modeling the time-dependent component of soil deformation due to consolidation effects.

Early analytical models for transient fluid diffusion through porous and deformable media have been developed from the pioneering works of Terzaghi [2] and Biot [3-6], who laid the mathematical foundations of the theory for linear elastic porous media under one- and three-dimensional settings, respectively. The general formulation of the theory of consolidation was well ahead of its time [7], and only after two decades since its 
development did similar developments take place on the general subject of continuum theory of mixtures (see [8] for a narrative on the historical development of the mixture theory, as well as [9-11] for some additional references), which applies to general multiphase media such as gas mixtures, fluid mixtures, bubbly liquids, suspensions, and alloys. With respect to the problem of soil consolidation, Biot's three-dimensional theory has been the basis of most subsequent works in geophysics, soil, and rock mechanics [12-14]. The theory has since been extended to include nonlinear and irreversible material responses [15-20], as well as the effects of large deformations [21-23] that could develop in deposits of highly compressible clays.

To date, extensions of the infinitesimal consolidation theory to the finite deformation regime have been formulated within the framework of hypoelasticity [21-26]. A hypoelastic formulation restricts the validity of the rate-constitutive equation to small elastic strains [27], and obscures a proper definition of the mean gradients and average volume changes necessary for imposing the mass conservation equation over a finite load increment [28]. Furthermore, a hypoelastic formulation requires the use of some non-unique measure of objective stress rate, such as the Jaumann stress rate which is known to behave poorly in simple shear [29].

Quite recently, an alternative formulation based on multiplicative plasticity has been proposed for analyzing the problem of elastoplastic consolidation at finite strain [28,30]. The formulation extends the ideas in [27] to two-phase soil-water continua, and now involves a constrained boundary-value problem in which the volumetric deformation of the solid matrix is constrained by the relative motion of the fluid phase. In addition to allowing for the development of large elastic strains, the new formulation also circumvents the rate issue in finite deformation analysis. An interesting by-product of the formulation presented in [28] is that for saturated soil media with incompressible solid grains and fluids, balance of energy suggests that Terzaghi's effective stress is the appropriate measure of stress for describing the constitutive response of the soil skeleton. Consequently, the formulation has the advantage of being able to accommodate a majority of the effective stress-based models developed in geotechnical engineering for describing the deformation behavior of compressible clays [31].

This paper builds upon the mathematical model for finite strain elastoplastic consolidation presented in [28] and casts the theory within the framework of nonlinear finite element analysis. Matrix forms of the variational equations are developed, and numerical examples are run to test the robustness of the finite element model. A central issue in elastoplastic consolidation analysis concerns the role played by the constitutive model particularly with respect to its capability to simulate plastic compaction and dilation. In this paper, we employ a critical state model originally proposed in [32], and later modified in [33-36] to accommodate large deformation effects. This model can replicate some of the most important features of compressible clay behaviors, such as plastic compaction and dilation as well as their associated hardening and softening responses. In [33] the model has been cast within the framework of multiplicative plasticity, which is the version of the model used in this paper to test the robustness of the proposed finite element consolidation model.

As for notations and symbols, bold-face letters denote matrices and vectors; the symbol '.' denote an inner product of two vectors (e.g. $\boldsymbol{a} \cdot \boldsymbol{b}=a_{i} b_{i}$ ), or a single contraction of adjacent indices of two tensors (e.g. $\boldsymbol{c} \cdot \boldsymbol{d}=c_{i j} d_{j k}$ ); the symbol ' $\because$ ' denotes an inner product of two second-order tensors (e.g. $\boldsymbol{c}: \boldsymbol{d}=\boldsymbol{c}_{i j} d_{i j}$ ), or a double contraction of adjacent indices of tensors of rank two and higher (e.g. $D: C=D_{I J K L} C_{K L}$ ).

\section{Field equations in variational form}

Let $\mathscr{B} \subset R^{n_{s d}}$ define a fluid-saturated simple soil body bounded by the surface $\partial \mathscr{B}$ in the reference configuration. Further, let $\phi$ be the solid phase motion and $\theta$ be the Kirchhoff pore water pressure. For future reference in this paper, let us recall the following results presented in [28].

In variational form, balance of linear momentum in the absence of inertia and rotational stresses reads

$$
G(\boldsymbol{\phi}, \theta, \boldsymbol{\eta})=\int_{\mathscr{B}}\left(\operatorname{grad} \boldsymbol{\eta}: \boldsymbol{\tau}-\boldsymbol{\theta} \operatorname{div} \boldsymbol{\eta}-\rho_{0} \boldsymbol{\eta} \cdot \boldsymbol{G}\right) \mathrm{d} V-\int_{\partial \dot{B} \boldsymbol{r}} \boldsymbol{\eta} \cdot \boldsymbol{t} \mathrm{d} A=0,
$$

where $\boldsymbol{\eta}$ is the displacement variation field. The variational form of balance of mass assuming incompressible solid grains and fluids is

$$
H(\phi, \theta, \psi)=\int_{B S}(\psi j-\operatorname{grad} \psi \cdot J \tilde{\boldsymbol{v}}) \mathrm{d} V-\int_{\dot{\partial} \mathscr{B} h} \psi Q \mathrm{~d} A=0
$$


where $\psi$ is the pore pressure variation field. In (2.1) and (2.2), $\boldsymbol{\tau}=J \boldsymbol{\sigma}$ is the symmetric Kirchhoff effective stress tensor obtained by multiplying the Cauchy effective stress tensor $\boldsymbol{\sigma}$ by the Jacobian $J$ of the solid phase motion; $\boldsymbol{G}$ is the vector of gravity accelerations; $\boldsymbol{t}$ is the prescribed traction vector on $\partial \mathscr{B}{ }^{t} \subset \partial \mathscr{B}$ reckoned with respect to the reference configuration; $\rho_{0}$ is a non-constant reference mass density of the soil mass; $j$ is the time derivative of $J ; \tilde{\boldsymbol{v}}$ is the relative velocity of flow per unit area of the deforming soil mass; $Q$ is the prescribed volumetric rate of flow per unit undeformed area across the boundary $\partial \mathscr{B}^{h} \subset \mathscr{B}$ ( $Q=0$ usually); grad is the spatial gradient operator; and div is the spatial divergence operator.

The sequence of temporal and spatial discretizations may be interchanged, and so this feature may be exploited to eliminate the rate term $j$ in (2.2) at the outset. To this end, we consider the following time-integrated variational equation $[15,16]$ :

$$
\begin{aligned}
H_{\Delta t}(\phi, \theta, \psi)= & \int_{\mathscr{B}} \frac{\psi}{\Delta t}\left(J_{n+1}-\sum_{m=1}^{k} \alpha_{m} J_{n+1-m}\right) \mathrm{d} V \\
& -\beta_{0} \int_{\mathscr{B}}\left[\beta(\operatorname{grad} \psi \cdot J \tilde{\boldsymbol{v}})_{n+1}+(1-\beta)(\operatorname{grad} \psi \cdot J \tilde{\boldsymbol{v}})_{n}\right] \mathrm{d} V \\
& -\beta_{0} \int_{\partial \mathscr{B}} \psi\left[\beta Q_{n+1}+(1-\beta) Q_{n}\right] \mathrm{d} A=0,
\end{aligned}
$$

where $\Delta t=t_{n+1}-t_{n}$; and $\beta, \beta_{0}$, and the $\alpha_{m}$ 's are time-integration parameters. The well-known trapezoidal family of methods is recovered from (2.3) by setting $k=1, \beta_{0}=1, \alpha_{1}=1$, and $\beta \in[0,1]$. If $\beta=1$ and $k \geqslant 1$, then we recover the family of unconditionally stable, $k$-order accurate $k$-step backward differentiation formula (BDF) methods $[15,16]$. In this paper we will consider (2.1) and the temporally discretized (2.3) for subsequent introduction of the spatial discretization functions.

Since $G$ and $H_{\Delta t}$ are both zero, their first variations $\delta G$ and $\delta H_{\Delta t}$ also must vanish. Setting $\delta G=0$ gives

$$
\begin{aligned}
\delta G= & \int_{\mathscr{B}} \operatorname{grad} \boldsymbol{\eta}:(\boldsymbol{c}+\boldsymbol{\tau} \oplus \mathbf{1}): \operatorname{grad} \delta \boldsymbol{u} \mathrm{d} V-\int_{\mathscr{B}}\left(\delta \theta \operatorname{div} \boldsymbol{\eta}-\theta \operatorname{grad}^{\mathrm{t}} \boldsymbol{\eta}: \operatorname{grad} \delta \boldsymbol{u}\right) \mathrm{d} V \\
& -\int_{\mathscr{B}} \rho_{\mathrm{w}} J \operatorname{div}(\delta \boldsymbol{u}) \boldsymbol{\eta} \cdot \boldsymbol{G} \mathrm{d} V-\int_{\partial \mathscr{B}} \boldsymbol{\eta} \cdot \delta \boldsymbol{t} \mathrm{d} A=0,
\end{aligned}
$$

where $\delta \boldsymbol{u}, \delta \theta$ and $\delta t$ are the respective variations of the displacement vector, Kirchhoff pore water pressure, and traction vector. The first integral in (2.4) contains the initial stress term $(\boldsymbol{\tau} \oplus \mathbf{1})_{i j k l}=\tau_{j l} \delta_{i k}$, with $(\mathbf{1})_{i j}=\delta_{i j}$ being the Kronecker delta, as well as the spatial tangential stiffness tensor $\boldsymbol{c}$; the third integral represents the variation of the (non-constant) reference mass density $\rho_{0}$ reflecting the amount of fluid with a constant mass density $\rho_{\mathrm{w}}$ that enters into or escapes from the soil matrix due to the variation of the Jacobian.

Setting $\delta H_{\Delta t}=0$ with $\Delta t$ fixed gives

$$
\begin{aligned}
\delta H_{\Delta t}= & \int_{\mathscr{B}} \frac{\psi}{\Delta t} J \operatorname{div} \delta \boldsymbol{u} \mathrm{d} V+\beta \beta_{0} \int_{\mathscr{B}} \operatorname{grad} \psi \cdot \frac{\boldsymbol{k}}{\rho_{\mathrm{w}} \mathrm{g}} \cdot \operatorname{grad} \delta \theta \mathrm{d} V \\
& -2 \beta \beta_{0} \int_{\mathscr{B}} \operatorname{grad} \psi \cdot \operatorname{symm}\left(\frac{\boldsymbol{k}}{\rho_{\mathrm{w}} \mathrm{g}} \cdot \operatorname{grad}^{\mathrm{t}} \delta \boldsymbol{u}\right) \cdot \operatorname{grad} \theta \mathrm{d} V \\
& -\beta \beta_{0} \int_{\mathscr{B}} \operatorname{grad} \psi \cdot[\operatorname{grad} \delta \boldsymbol{u}-(\operatorname{div} \delta \boldsymbol{u}) \mathbf{1}] \cdot \boldsymbol{k} \cdot \frac{\boldsymbol{G}}{\mathrm{g}} J \mathrm{~d} V-\beta \beta_{0} \int_{\partial \mathscr{B}} \psi \delta Q \mathrm{~d} A=0,
\end{aligned}
$$

where $\delta Q$ is the variation of the fluid flux $Q, \mathrm{~g}$ is the gravity acceleration constant, and $\boldsymbol{G}$ is the vector of gravity accelerations (note: $\|\boldsymbol{G}\| \equiv \mathrm{g}$ ). The second, third, and fourth integrals contain the second-order spatial permeability tensor $\boldsymbol{k}$ (assumed constant) obtained by generalizing Darcy's law to problems in two and three dimensions.

\section{Matrix equations}

The finite element matrix equations can be derived following standard lines. The idea is to introduce two possibly distinct spatial interpolation function matrices $N^{\phi}(x)$ and $N^{\theta}(x)$ for approximating the solid phase motion $\phi$ and the pore pressure field $\theta$. 
Let the solid phase motion $\phi$ be approximated by the spatial displacement field $\boldsymbol{u}^{h}(\boldsymbol{x}) \in R^{n_{s d l}}$. In matrix form, we have

$$
u^{h}(x)=N^{\phi}(x) d+N_{g}^{\phi}(x) d_{g},
$$

where $d \in R^{\mathrm{NQ}}$ is the unknown nodal solid displacement vector and $\boldsymbol{d}_{g}$ is the vector of prescribed nodal solid displacements. Similarly, let the spatial Kirchhoff pore pressure field $\theta$ be approximated by the function $\theta^{h}(x) \in R^{1}$. In matrix form, we have

$$
\theta^{h}(\boldsymbol{x})=\boldsymbol{N}^{\theta}(\boldsymbol{x}) \boldsymbol{\theta}+\boldsymbol{N}_{r}^{\theta}(\boldsymbol{x}) \boldsymbol{\theta}_{r},
$$

where $\boldsymbol{\theta} \in R^{\mathrm{NP}}$ is the unknown nodal Kirchhoff pore pressure vector and $\boldsymbol{\theta}_{r}$ is the vector of prescribed nodal Kirchhoff pore pressures. The weighting functions $\boldsymbol{\eta}$ and $\psi$ may be approximated in a similar fashion in terms of their nodal values $\tilde{\boldsymbol{\eta}}$ and $\tilde{\boldsymbol{\psi}}$ as follows:

$$
\boldsymbol{\eta}^{h}(x)=N^{\phi}(x) \tilde{\boldsymbol{\eta}} ; \quad \psi^{h}(x)=\boldsymbol{N}^{\theta}(x) \tilde{\psi}
$$

where $\tilde{\boldsymbol{\eta}} \in R^{\mathrm{NQ}}$ and $\tilde{\boldsymbol{\psi}} \in R^{\mathrm{NP}}$. With these preliminaries in hand, we obtain the following results.

PROPOSITION 1. Let the weighting function $\boldsymbol{\eta}$ be approximated by any arbitrary nodal values $\tilde{\boldsymbol{\eta}} \in R^{\mathrm{NQ}}$ via $(3.3)_{1}$; then the finite element equation for balance of linear momentum in the absence of inertia and rotational stresses may be written as

$$
G^{h}(\phi, \theta, \tilde{\boldsymbol{\eta}})=\tilde{\boldsymbol{\eta}}^{\mathrm{t}}\left[\boldsymbol{N}^{s}(\boldsymbol{d})+\boldsymbol{N}^{w}(\boldsymbol{\theta})-\boldsymbol{F}_{\mathrm{EXT}}\right]=0,
$$

where

$$
\begin{aligned}
& \boldsymbol{N}^{s}(\boldsymbol{d})=\int_{\mathscr{B}} \boldsymbol{B}^{\mathrm{t}}\{\boldsymbol{\tau}\} \mathrm{d} V \\
& \boldsymbol{N}^{w}(\boldsymbol{\theta})=-\int_{\mathscr{B}} \boldsymbol{b}^{\mathrm{t}}\left(\boldsymbol{N}^{\theta} \boldsymbol{\theta}+\boldsymbol{N}_{r}^{\theta} \boldsymbol{\theta}_{r}\right) \mathrm{d} V \\
& \boldsymbol{F}_{\mathrm{EXT}}=\int_{\mathscr{B}} \rho_{0} \boldsymbol{N}^{\phi \mathrm{t}} \boldsymbol{G} \mathrm{d} V+\int_{\dot{\partial} \mathscr{B}} \boldsymbol{N}^{\phi \mathrm{t}} \mathbf{t} \mathrm{d} A
\end{aligned}
$$

and $\{\tau\}=\left\{\tau_{11}, \tau_{22}, \tau_{33}, \tau_{12}, \tau_{23}, \tau_{13}\right\}^{t}$ for $n_{s d}=3$.

PROOF. Define $\boldsymbol{B}$ as the usual (spatial) strain-displacement transformation matrix with a structure

$$
\boldsymbol{B}=\left[\boldsymbol{B}_{1}, \boldsymbol{B}_{2}, \ldots, \boldsymbol{B}_{\mathrm{NQ}}\right] .
$$

For example, for $n_{s d}=3, \boldsymbol{B}$ is of dimension $6 \times 3 \mathrm{NQ}$. Observing that $\boldsymbol{\tau}$ is symmetric and expanding terms, we have $\tilde{\boldsymbol{\eta}}^{\mathbf{t}} \boldsymbol{B}^{\mathrm{t}}\{\boldsymbol{\tau}\}=\operatorname{grad} \boldsymbol{\eta}: \boldsymbol{\tau}$, so that (3.5a) produces the first integral term in (2.1). Next, define $\boldsymbol{b}=\{\mathbf{1}\} \boldsymbol{B}^{\mathrm{B}}$, where $\{\mathbf{1}\}=\{1,1,1,0,0,0\}^{t}$ and $\boldsymbol{b}$ is of dimension $1 \times 3 \mathrm{NQ}$ for $n_{s d}=3$. It follows that $\boldsymbol{b} \tilde{\boldsymbol{\eta}}=\tilde{\boldsymbol{\eta}}^{\mathbf{b}} \boldsymbol{b} \equiv \operatorname{div} \boldsymbol{\eta}$, so that (3.5b) produces the second integral term in (2.1). The third and fourth integral terms in (2.1) may be obtained from $(3.5 \mathrm{c})$ following similar lines.

PROPOSITION 2. Let the weighting function $\psi$ be approximated by any arbitrary nodal values $\tilde{\psi} \in R^{\mathrm{NP}}$ via $(3.3)_{2}$; then the finite element equation for balance of mass assuming incompressible solid grains and fluids may be written as

$$
-\Delta t H_{\Delta t}^{h}(\phi, \theta, \tilde{\psi})=\tilde{\psi}^{t}\left[J(\boldsymbol{d})+\beta_{0} \Delta t \boldsymbol{\Phi}(\boldsymbol{\theta})+\beta_{0} \Delta t \boldsymbol{H}_{\mathrm{EXT}}\right]=0
$$

where 


$$
\begin{aligned}
& \boldsymbol{J}(\boldsymbol{d})=-\int_{\mathscr{B}} \boldsymbol{N}^{\theta 1}\left(J_{n+1}-\sum_{m=1}^{k} \alpha_{m} J_{n+1-m}\right) \mathrm{d} V \\
& \boldsymbol{\Phi}(\boldsymbol{\theta})=\beta \int_{\mathscr{B}} \boldsymbol{E}^{\mathrm{t}} J_{n+1} \tilde{\boldsymbol{v}}_{n+1} \mathrm{~d} V+(1-\beta) \int_{\mathscr{B}} \boldsymbol{E}^{\mathrm{t}} \boldsymbol{J}_{n} J_{n} \tilde{\boldsymbol{v}}_{n} \mathrm{~d} V \\
& \boldsymbol{H}_{\mathrm{EXT}}=\int_{\mathscr{B}} \boldsymbol{N}^{\theta \mathrm{t}}\left[\beta Q_{n+1}+(1-\beta) Q_{n}\right] \mathrm{d} A
\end{aligned}
$$

and $f_{n}=\partial x / \partial x_{n}$ is the local deformation gradient reckoned with respect to the configuration at time $t_{n}$.

PROOF. From (3.3) $)_{2}$, we have $N^{\theta} \tilde{\psi}=\tilde{\psi}^{t} N^{\theta t} \equiv \psi^{h}$, and so (3.7a) and (3.7c) produce the first and the third integral terms in (2.3), respectively. Next, define $\boldsymbol{E}$ as the gradient-pressure transformation matrix with a structure

$$
\boldsymbol{E}=\left[\operatorname{grad} N_{1}^{\theta}, \operatorname{grad} N_{2}^{\theta}, \ldots, \operatorname{grad} N_{\mathrm{NP}}^{\theta}\right] .
$$

For example, for $n_{s d}=3, \boldsymbol{E}$ is of dimension $3 \times$ NP. Hence, $\boldsymbol{E} \tilde{\boldsymbol{\eta}}=\operatorname{grad} \boldsymbol{\eta}$ and $\boldsymbol{f}_{n}^{\mathrm{t}} \boldsymbol{E} \tilde{\boldsymbol{\eta}}=\operatorname{grad}_{n} \boldsymbol{\eta}$, and so (3.7b) produces the second integral term in (2.3).

The approximations stated above can also be used to express the first variations of $G^{h}$ and $H_{\Delta t}^{h}$ in terms of matrices. Following [28], we assume a condition of dead loading and impose additional conditions of dead external traction $(\delta t=\mathbf{0})$ and dead external flux $(\delta Q=0)$. The first variations of $G^{h}$ and $H_{\Delta t}^{h}$ then become linear functions of the first variations of $\boldsymbol{d}$ and $\boldsymbol{\theta}$.

PROPOSITION 3. Let the weighting function $\boldsymbol{\eta}$ be approximated by any arbitrary nodal values $\tilde{\boldsymbol{\eta}} \in R^{\mathrm{NQ}}$ via (3.3) $)_{1}$, then the finite element equation for the first variation of $G^{h}$ may be written as

$$
\delta G^{h}(\phi, \theta, \tilde{\boldsymbol{\eta}})=\tilde{\boldsymbol{\eta}}^{\mathrm{t}}\left[\boldsymbol{K}_{\phi \phi} \delta \boldsymbol{d}+\boldsymbol{K}_{\phi \theta} \delta \boldsymbol{\theta}\right],
$$

where

$$
\begin{aligned}
& \boldsymbol{K}_{\phi \phi}=\int_{\mathscr{B}}\left(\boldsymbol{B}^{\prime} \boldsymbol{C B}+\tilde{\boldsymbol{B}}^{1} \boldsymbol{T} \tilde{\boldsymbol{B}}+\tilde{\boldsymbol{B}}^{\prime} \boldsymbol{I}_{\theta} \tilde{\boldsymbol{B}}-\rho_{\mathrm{w}} J \boldsymbol{N}^{\theta \mathrm{t}} \boldsymbol{G} \boldsymbol{b}\right) \mathrm{d} V \\
& \boldsymbol{K}_{\phi \theta}=-\int_{\mathscr{B}} \boldsymbol{b}^{\mathrm{t}} \boldsymbol{N}^{\theta} \mathrm{d} V,
\end{aligned}
$$

and $\delta \boldsymbol{d}, \delta \boldsymbol{\theta}$ are the first variations of $\boldsymbol{d}$ and $\boldsymbol{\theta}$, respectively.

PROOF. Arrange the elements of the fourth-order tensor $c$ into a material stiffness matrix $C$ (for $n_{s d}=3, C$ is of size $6 \times 6$ ) according to the procedure outlined in [37]. A simple expansion of the scalar product term $\tilde{\boldsymbol{\eta}}^{\mathrm{t}} \boldsymbol{B}^{\prime} \boldsymbol{C} \boldsymbol{B} \delta \boldsymbol{d}$ (note: $\delta \boldsymbol{d}_{g}=\mathbf{0}$ ) then yields the equivalent expression for $\operatorname{grad} \boldsymbol{\eta}^{h}: \boldsymbol{c}: \operatorname{grad} \delta \boldsymbol{u}^{h}$ in (2.4). This is the material stiffness contribution to the coefficient matrix.

Next, define the matrix

$$
\tilde{\boldsymbol{B}}=\left[\begin{array}{c}
\boldsymbol{B}^{\mathrm{s}} \\
\boldsymbol{B}^{\text {sk }}
\end{array}\right]=\left[\begin{array}{cccc}
\boldsymbol{B}_{1} & \boldsymbol{B}_{2} & \cdots & \boldsymbol{B}_{\mathrm{NQ}} \\
\boldsymbol{B}_{1}^{\text {sk }} & \boldsymbol{B}_{2}^{\text {sk }} & \cdots & \boldsymbol{B}_{\mathrm{NQ}}^{\text {sk }}
\end{array}\right]
$$

where $\boldsymbol{B}^{\text {sk }}$ is the skew component of $\tilde{\boldsymbol{B}}$ representing the rotational effects. For example, for $n_{s d}=3$ the matrix $\boldsymbol{B}^{\mathrm{sk}}$ is of dimension $3 \times 3 \mathrm{NQ}$, and $\tilde{\boldsymbol{B}}$ is of dimension $9 \times 3 \mathrm{NQ}$. Further, let the elements of the tensor $\boldsymbol{\tau}$ be assembled in the matrix $\boldsymbol{T}$ according to the procedure outlined in [38]. For $n_{s d}=3$, the matrix $\boldsymbol{T}$ is of dimension $9 \times 9$, and is always symmetric. A simple expansion of the scalar product term $\tilde{\boldsymbol{\eta}}^{t} \tilde{\boldsymbol{B}}^{\mathrm{t}} \boldsymbol{T} \tilde{\boldsymbol{B}} \boldsymbol{\delta} \boldsymbol{d}$ then yields the equivalent expression for $\operatorname{grad} \boldsymbol{\eta}^{h}: \boldsymbol{\tau} \oplus \mathbf{1}: \operatorname{grad} \delta \boldsymbol{u}^{h}$ in (2.4). This is the initial stress contribution to the coefficient matrix.

Finally, define a diagonal matrix $\boldsymbol{I}_{\theta}$ such that for $n_{s d}=3, \boldsymbol{I}_{\theta}$ is of dimension $9 \times 9$, and $\left(\boldsymbol{I}_{\theta}\right)_{i i}=\theta^{h}$ for $i=1,2,3 ; \quad\left(I_{\theta}\right)_{i i}=\theta^{h} / 2$ for $i=4,5,6$; and $\left(I_{\theta}\right)_{i i}=-\theta^{h} / 2$ for $i=7,8,9$. Expanding the product term $\tilde{\boldsymbol{\eta}}^{\prime} \tilde{\boldsymbol{B}}^{\mathrm{t}} \boldsymbol{I}_{\theta} \tilde{\boldsymbol{B}} \delta \boldsymbol{d}$ then results in the equivalent expression for $\theta^{h} \operatorname{grad}^{\prime} \boldsymbol{\eta}^{h}: \operatorname{grad} \delta \boldsymbol{u}^{h}$. This produces the initial pore 
pressure contribution to the coefficient matrix. The remaining terms can be proved using the identities $\boldsymbol{b} \delta \boldsymbol{d}=\operatorname{div}\left(\delta \boldsymbol{u}^{h}\right)$ and $\boldsymbol{b} \tilde{\boldsymbol{\eta}}=\operatorname{div}\left(\boldsymbol{\eta}^{h}\right)$, together with the essential boundary condition $\delta \boldsymbol{\theta}_{r}=\mathbf{0}$.

PROPOSITION 4. Let the weighting function $\psi$ be approximated by any arbitrary nodal values $\tilde{\psi} \in R^{\mathrm{NP}}$ via (3.3) $)_{2}$, then the finite element equation for the first variation of $H_{\Delta t}^{h}$ may be written as

$$
-\Delta t \delta H_{\Delta t}^{h}(\phi, \theta, \tilde{\boldsymbol{\psi}})=\tilde{\boldsymbol{\psi}}^{\mathrm{t}}\left[\boldsymbol{K}_{\theta \phi} \delta \boldsymbol{d}+\boldsymbol{K}_{\theta \theta} \delta \boldsymbol{\theta}\right],
$$

where

$$
\begin{aligned}
& \boldsymbol{K}_{\theta \phi}=-\int_{\partial B} J N^{\theta t} \boldsymbol{b} \mathrm{d} V+\beta \beta_{0} \Delta t \int_{\mathscr{B}}\left(\frac{1}{\rho_{\mathrm{w}} \mathrm{g}} \boldsymbol{E}^{\mathrm{t}} \boldsymbol{A} \tilde{\boldsymbol{B}}-J \boldsymbol{E}^{\mathrm{t}} \boldsymbol{W} \tilde{\boldsymbol{B}}\right) \mathrm{d} V \\
& \boldsymbol{K}_{\theta \theta}=-\frac{\beta \beta_{0} \Delta t}{\rho_{\mathrm{w}} \mathrm{g}} \int_{\not B} \boldsymbol{E}^{\mathrm{t}} \boldsymbol{k} \boldsymbol{E} \mathrm{d} V,
\end{aligned}
$$

and $\delta \boldsymbol{d}, \delta \boldsymbol{\theta}$ are the first variations of $\boldsymbol{d}$ and $\boldsymbol{\theta}$, respectively.

PROOF. The matrix forms for the first two integrals in (2.5) are trivial. With respect to the third integral in (2.5), which arises from geometric nonlinearity, the following identity can be obtained by direct expansion:

$$
2 \operatorname{grad} \psi^{h} \cdot \operatorname{symm}\left[\boldsymbol{k} \cdot \operatorname{grad}^{\mathrm{t}}\left(\delta u^{h}\right)\right] \cdot \operatorname{grad} \theta=\tilde{\boldsymbol{\psi}}^{\mathrm{t}} \underbrace{\boldsymbol{E}^{\mathrm{t}} \boldsymbol{A} \tilde{\boldsymbol{B}}}_{\text {see (3.11a) }} \delta \boldsymbol{d}
$$

where

$$
\boldsymbol{A}=\left[\boldsymbol{A}_{1}, \boldsymbol{A}_{2}, \boldsymbol{A}_{3}\right]
$$

and (for $n_{s d}=3$ )

$$
\begin{aligned}
& \boldsymbol{A}_{1}=\left[\begin{array}{lll}
k_{11} \theta_{, 1} & k_{12} \theta_{.2} & k_{13} \theta_{, 3} \\
k_{21} \theta_{.1} & k_{22} \theta_{.2} & k_{23} \theta_{3} \\
k_{31} \theta_{.1} & k_{32} \theta_{.2} & k_{33} \theta_{, 3}
\end{array}\right]+\left[\begin{array}{ccc}
\hat{v}_{1} & 0 & 0 \\
0 & \hat{v}_{2} & 0 \\
0 & 0 & \hat{v}_{3}
\end{array}\right] \\
& 2 \boldsymbol{A}_{2}=\left[\begin{array}{lll}
k_{11} \theta_{2}+k_{12} \theta_{11} & k_{12} \theta_{3}+k_{13} \theta_{, 2} & k_{11} \theta_{3}+k_{13} \theta_{, 1} \\
k_{21} \theta_{, 2}+k_{22} \theta_{, 1} & k_{22} \theta_{33}+k_{23} \theta_{, 2} & k_{21} \theta_{3}+k_{23} \theta_{, 1} \\
k_{31} \theta_{, 2}+k_{32} \theta_{, 1} & k_{32} \theta_{, 3}+k_{33} \theta_{, 2} & k_{31} \theta_{, 3}+k_{33} \theta_{, 1}
\end{array}\right]+\left[\begin{array}{ccc}
\hat{v}_{2} & 0 & \hat{v}_{3} \\
\hat{v}_{1} & \hat{v}_{3} & 0 \\
0 & \hat{v}_{2} & \hat{v}_{1}
\end{array}\right] \\
& \mathbf{2 A}_{3}=\left[\begin{array}{lll}
k_{12} \theta_{.1}-k_{11} \theta_{.2} & k_{13} \theta_{.2}-k_{12} \theta_{, 3} & k_{13} \theta_{, 1}-k_{11} \theta_{3} \\
k_{22} \theta_{, 1}-k_{21} \theta_{.2} & k_{23} \theta_{.2}-k_{22} \theta_{3} & k_{23} \theta_{, 1}-k_{21} \theta_{, 3} \\
k_{32} \theta_{, 1}-k_{31} \theta_{, 2} & k_{33} \theta_{, 2}-k_{32} \theta_{, 3} & k_{33} \theta_{, 1}-k_{31} \theta_{, 3}
\end{array}\right]+\left[\begin{array}{ccc}
\hat{v}_{2} & 0 & \hat{v}_{3} \\
-\hat{v}_{1} & \hat{v}_{3} & 0 \\
0 & -\hat{v}_{2} & -\hat{v}_{1}
\end{array}\right] \text {, }
\end{aligned}
$$

with $\hat{v}_{i}=k_{i j} \theta_{. j}$ (sum on $j=1,2,3$ ). For $n_{s d}=3$, the matrix $\boldsymbol{A}$ is of dimension $3 \times 9$.

With respect to the fourth integral in (2.5), which also arises from geometric nonlinearity, the following identity can be obtained by direct expansion:

$$
\operatorname{grad} \psi^{h} \cdot\left[\operatorname{grad}\left(\delta \boldsymbol{u}^{h}\right)-\operatorname{div}\left(\delta \boldsymbol{u}^{h}\right) \mathbf{1}\right] \cdot \boldsymbol{k} \cdot \frac{\boldsymbol{G}}{\mathrm{g}}=\tilde{\boldsymbol{\psi}}^{\mathrm{t}} \underbrace{\boldsymbol{E}^{\mathbf{t}} \boldsymbol{W} \tilde{\boldsymbol{B}}}_{\text {see }(3.11 \mathrm{a})} \delta \boldsymbol{d}
$$

where

$$
\boldsymbol{W}=\left[\boldsymbol{W}_{1}, \boldsymbol{W}_{2}, \boldsymbol{W}_{3}\right]
$$

and 


$$
\begin{aligned}
& \boldsymbol{W}_{1}=-\frac{1}{\mathrm{~g}}\left[\begin{array}{ccc}
0 & k_{1 i} G_{i} & k_{1 i} G_{i} \\
k_{2 i} G_{i} & 0 & k_{2 i} G_{i} \\
k_{3 i} G_{i} & k_{3 i} G_{i} & 0
\end{array}\right] ; \quad 2 \boldsymbol{W}_{2}=\frac{1}{\mathrm{~g}}\left[\begin{array}{ccc}
k_{2 i} G_{i} & 0 & k_{3 i} G_{i} \\
k_{1 i} G_{i} & k_{3 i} G_{i} & 0 \\
0 & k_{2 i} G_{i} & k_{1 i} G_{i}
\end{array}\right] ; \\
& 2 \boldsymbol{W}_{3}=\frac{1}{\mathrm{~g}}\left[\begin{array}{ccc}
k_{2 i} G_{i} & 0 & k_{3 i} G_{i} \\
-k_{1 i} G_{i} & k_{3 i} G_{i} & 0 \\
0 & -k_{2 i} G_{i} & -k_{1 i} G_{i}
\end{array}\right],
\end{aligned}
$$

with a summation implied over the index $i=1,2,3$. For $n_{s d}=3$, the matrix $\boldsymbol{W}$ also is of dimension $3 \times 9$.

Since $\tilde{\eta}$ and $\tilde{\psi}$ are both arbitrary, the conditions stated in (3.4) and (3.6) can be satisfied by the following (coupled) vector equations:

\section{Balance of momentum:}

$$
\boldsymbol{r}_{\phi}(d, \boldsymbol{\theta})=N^{s}(d)+N^{w}(\boldsymbol{\theta})-\boldsymbol{F}_{\mathrm{EXT}}=\mathbf{0} .
$$

Balance of mass:

$$
\boldsymbol{r}_{\theta}(\boldsymbol{d}, \boldsymbol{\theta})=\boldsymbol{J}(\boldsymbol{d})+\beta_{0} \Delta t \boldsymbol{\Phi}(\boldsymbol{\theta})+\beta_{0} \Delta t \boldsymbol{H}_{\mathrm{EXT}}=\mathbf{0} .
$$

For numerical analysis, the problem boils down to determining the configurations defined by the nodal values $\boldsymbol{d}$ and $\boldsymbol{\theta}$ at which (3.12) and (3.13) are simultaneously satisfied.

If $\boldsymbol{r}=\left\{\boldsymbol{r}_{\phi}, \boldsymbol{r}_{\theta}\right\}^{1} \neq \mathbf{0}$ for some trial configurations $\boldsymbol{d}^{k}$ and $\boldsymbol{\theta}^{k}$, the numerical solution may be iterated via Newton's method. However, the iteration generally requires the use of a consistent tangent operator which, for the problem at hand, can simply be assembled from the previously defined coefficient matrices as

$$
\boldsymbol{K}=\left[\begin{array}{ll}
\boldsymbol{K}_{\phi \phi} & \boldsymbol{K}_{\phi \theta} \\
\boldsymbol{K}_{\theta \phi} & \boldsymbol{K}_{\theta \theta}
\end{array}\right] .
$$

In general, the matrix $\boldsymbol{K}$ is non-symmetric and indefinite.

The lack of symmetry of $\boldsymbol{K}$ is a consequence of solving a non-symmetric consolidation problem. However, there are conditions which result in a symmetric $\boldsymbol{K}$ even if the problem of consolidation is inherently a non-symmetric one. Obviously, $\boldsymbol{K}$ being symmetric requires that $\boldsymbol{K}_{\theta \theta}=\boldsymbol{K}_{\theta \theta}^{\prime}$, which is true if and only if the permeability tensor $\boldsymbol{k}$ is symmetric. Furthermore, for small strain analysis the Jacobian $J$ is identically equal to unity, while the second integral in (3.11a) vanishes identically since it originally arises from geometric nonlinearity (see [28]). Thus, for this condition, $\boldsymbol{K}_{\theta \phi}=\boldsymbol{K}_{\phi \theta}^{t}$. Under the same setting imposed by the assumption of small strains, the last term in the integral of (3.9a) also vanishes, since this term is simply the linearization of the constant Jacobian. Thus, under the assumption of small strains, $\boldsymbol{K}_{\phi \phi}=\boldsymbol{K}_{\phi \phi}^{t}$ provided that $\boldsymbol{C}$ is symmetric (see [17]).

\section{Numerical examples}

This section demonstrates the significance of large deformation on the consolidation response of compressible clay foundations. The examples include one- and two-dimensional (plane strain) consolidation employing mixed finite elements which combine a biquadratic 9-node displacement interpolation with a bilinear 4-node pore pressure interpolation. Time integration is carried out by the one-step, first-order accurate, unconditionally stable backward difference scheme obtained by setting $k=1$, and $\beta_{0}=\beta=\alpha_{1}=1$ in (2.3). The analyses were run in double precision using a FE code called SPIN2D [16].

\subsection{One-dimensional hyperelastic consolidation}

Here, we consider an initially stress-free hyperelastic porous soil skeleton described by a free energy function $\Psi$ that is quadratic in the principal elastic logarithmic stretches [39]. The expression for $\Psi$ in terms of the Lamé parameters $\lambda$ and $\mu$ takes the form 


$$
\Psi=\frac{1}{2} \lambda\left[\varepsilon_{1}^{e}+\varepsilon_{2}^{e}+\varepsilon_{3}^{e}\right]^{2}+\mu\left[\left(\varepsilon_{1}^{e}\right)^{2}+\left(\varepsilon_{2}^{e}\right)^{2}+\left(\varepsilon_{3}^{e}\right)^{2}\right],
$$

where $\varepsilon_{A}^{e}=\ln \left(\lambda_{A}^{e}\right), A=1,2,3$, are the elastic logarithmic principal stretches. The assumed values of the material parameters are $\lambda=57.7 \mathrm{kPa}$ and $\mu=38.5 \mathrm{kPa}$ (equivalent to Young's modulus of $E=100 \mathrm{kPa}$ and Poisson's ratio of $\nu=0.3$ ).

The FE mesh is represented by a column of 10 mixed elements shown in Fig. 1. The mesh assumes an impervious bottom base that is fixed with respect to displacements, zero horizontal displacements on the vertical sides, and zero excess pore pressures on top. The vertical permeability is assumed to have a value of $k_{v}=8.64 \times 10^{-4} \mathrm{~m} /$ day; unit weight of water to $\rho_{\mathrm{w}} \mathrm{g}=10 \mathrm{kN} / \mathrm{m}^{3}$. For purposes of normalizing the results, it is useful to utilize the constrained modulus as [40] $D=\lambda+2 \mu=134.7 \mathrm{kPa}$; the coefficient of consolidation can then be calculated as $c_{\mathrm{v}}=k_{\mathrm{v}} D /\left(\rho_{\mathrm{w}} \mathrm{g}\right)=1.16 \times 10^{-2} \mathrm{~m}^{2} /$ day, while the normalized time factor is calculated as $T=c_{\mathrm{v}} t / H_{0}^{2}$, where $H_{0}$ is the initial thickness of the soil column (see [2]). During the consolidation stage, the time steps are increased according to the equation $\Delta t_{n+1}=1.5 \Delta t_{n}$. This results in nearly equally spaced data points when the time-history responses are plotted on the logarithmic time axis. Excess pore pressures are generated by applying a vertical downward Cauchy load of $\Delta w^{\text {(Cauchy) }}=-90 \mathrm{kPa}$ instantaneously at the top of the soil column, producing the initial pore pressure isochrone also shown in Fig. 1.

Fig. 2 shows a comparison of the variations with respect to time of the fluid potential $\Pi=\Pi^{\theta}+\Pi^{e}=$ $\theta /\left(J \rho_{\mathrm{w}} \mathrm{g}\right)+x_{2}$ at a Gauss point $A$ near the impervious base initially situated at a distance of $4.894 \mathrm{~m}$ from the top of the undeformed soil column. Here, the potential $\Pi$ takes the physical meaning of being the total hydraulic head at this particular Gauss point [41]. The small strain solution shown in Fig. 2 is generated analytically from the one-dimensional linear consolidation model of Terzaghi [2]. Prior to consolidation, the fluid potentials predicted by the small strain and the finite deformation models are the same and are equal to $14 \mathrm{~m}$, of which $9 \mathrm{~m}$ represents the transient part produced by the $90 \mathrm{kPa}$ imposed vertical load. Note in Fig. 2 that whereas the fluid potential predicted by the Terzaghi solution decays to the initial steady-state value of $\Pi^{(\mathrm{smatl})}=5 \mathrm{~m}$ since the height of the soil column remains essentially the same at $5 \mathrm{~m}$ due to the small strain assumption, the finite deformation solution approaches a steady-state value of $\Pi^{\text {(finite) }}=3.24 \mathrm{~m}$ representing the final compressed height of the soil column.

The validity of the finite deformation solution can be checked from the following simple manual calculations. For a one-dimensional constrained compression the Jacobian $J$ at steady-state condition can be calculated from the ratio of the final to initial column heights. Thus, $J=3.24 / 5=0.648$, and is constant throughout the height of the soil column. The final Kirchhoff effective vertical stress is equal to $\Delta w^{\text {(Kirchhoff) }}=J \Delta w^{(\text {Cauchy })}=$ $-58.32 \mathrm{kPa}$, which is also distributed uniformly throughout the height of the soil column at steady-state condition. Since the elastic constitutive equation is expressed in terms of the Kirchhoff stresses, we have

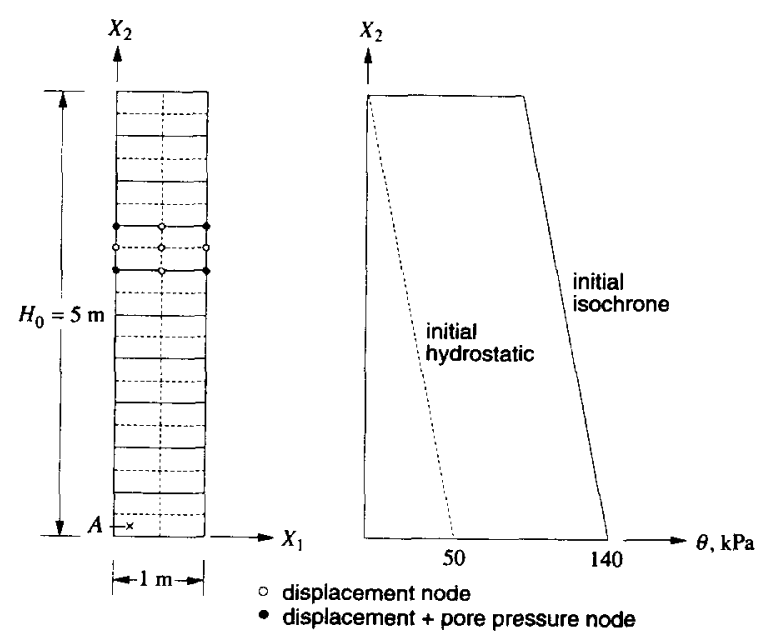

Fig. 1. Finite element mesh and initial pore water pressures for one-dimensional consolidation problem.

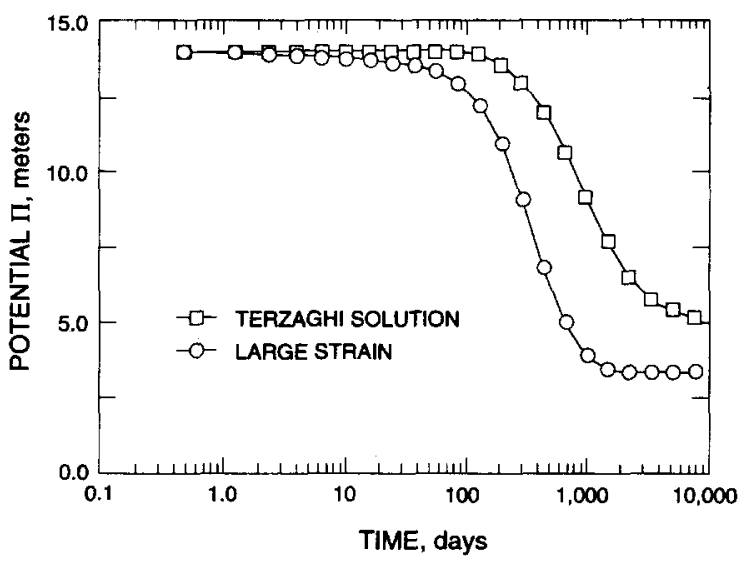

Fig. 2. One-dimensional hyperelastic consolidation: variation of total potential at Gauss point $A$ with time. 
$\Delta w^{(\text {Kirchhoff })}=D \varepsilon_{\mathrm{v}}^{e}$. This gives a uniform vertical elastic principal logarithmic stretch of $\varepsilon_{\mathrm{v}}^{e}=-0.433$. The corresponding vertical elastic principal stretch is $\lambda_{\mathrm{v}}^{e}=\exp \left(\varepsilon_{\mathrm{v}}^{e}\right)=0.648$, which checks with the computed steady-state value of the Jacobian $J$.

Fig. 3 shows the isochrones of Cauchy pore pressures plotted for different values of the equivalent time factor $T$. The Cauchy pore pressures were calculated as $\varphi=\vartheta / J$ at the Gauss points by interpolating the Kirchhoff nodal pore pressures provided by the global solution, and then dividing them by the Jacobians computed at the Gauss points (the Cauchy pore pressures cannot be evaluated at the nodes since the values of the Jacobians are not available at the nodes). Observe that the isochrones predicted by the finite deformation model move spatially as a result of the large deformation effect. For comparison purposes, the isochrones computed from the Terzaghi model are also plotted in Fig. 3.

\subsection{One-dimensional hyperelastic-plastic consolidation}

Next, we repeat the analysis of the previous section but now utilize a widely used critical state constitutive model for soils - the modified Cam-Clay plasticity model. With reference to finite deformation analysis, we are particularly interested in a problem where the domain of interest has no characteristic initial stress-free configuration relative to which the current configuration may be referenced. An example of such a situation is a soil deposit initially subjected to its own dead weight, where removing the gravity load does not necessarily lead to a meaningful initial stress-free configuration since soils are deposited in nature by a physical process of sedimentation. The example described below illustrates a procedure that may be used to establish a reference configuration that is not stress-free.

The initialization phase to establish an initially stressed reference configuration requires applying the soil's self-weight and determining the internal stresses that balance this load. Here, a small-strain analysis is carried out so that the internal stresses generated by the solution take on a Cauchy definition. Once the gravity loads have been imposed, the displacements may be reinitialized to zero prior to the beginning of the consolidation analysis. Since the post-gravity configuration is the reference configuration, the Jacobian takes on the initial value $J=1$ prior to the consolidation analysis.

Let us now describe an elasto-plastic constitutive model in which the elastic component shows the soil response being dependent on the values of the internal stresses. For clays, we consider a class of stored energy functions of the form

$$
\Psi\left(\varepsilon_{\mathrm{v}}^{\mathrm{e}}, \varepsilon_{\mathrm{s}}^{\mathrm{e}}\right)=p_{0} \tilde{\kappa} \exp \left(\frac{\varepsilon_{\mathrm{v}}^{\mathrm{e}}-\varepsilon_{\mathrm{v} 0}^{\mathrm{e}}}{\tilde{\kappa}}\right)+\frac{3}{2} \mu^{\mathrm{e}} \varepsilon_{\mathrm{s}}^{\mathrm{e} 2}
$$

where $\mu^{\mathrm{e}}$ is the elastic shear modulus defined by the expression

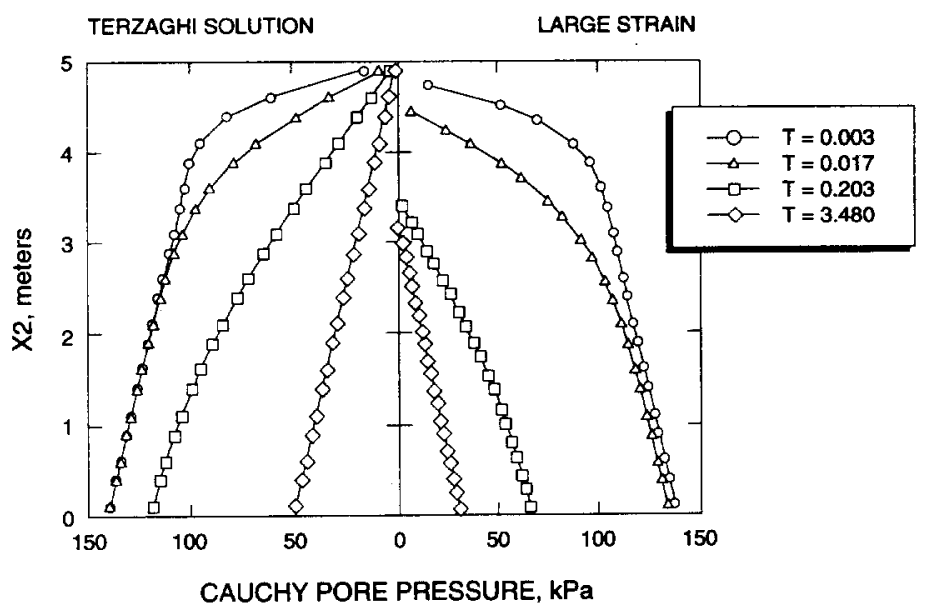

Fig. 3. One-dimensional hyperelastic consolidation: isochrones of constant Cauchy pore pressures. 


$$
\mu^{\mathrm{e}}=\mu_{0}+\alpha p_{0} \exp \left(\frac{\varepsilon_{\mathrm{v}}^{\mathrm{e}}-\varepsilon_{\mathrm{v} 0}^{\mathrm{e}}}{\tilde{\kappa}}\right)
$$

$\varepsilon_{\mathrm{v}}^{\mathrm{e}}$ and $\varepsilon_{\mathrm{s}}^{\mathrm{e}}$ are the elastic volumetric and deviatoric strain invariants, respectively; $p_{0}$ and $\varepsilon_{\mathrm{v} 0}^{\mathrm{e}}$ are reference parameters; and $\tilde{\kappa}$ is an elastic compressibility index. The stored energy function described in (4.2) results in an elasticity model where the elastic bulk modulus varies linearly with the effective mean normal stress [42]. By setting $\alpha=0$ the elastic shear modulus $\mu^{\mathrm{e}}$ can be approximated by a constant value $\mu_{0}>0$, while setting $\mu_{0}=0$ and $\alpha>0$ results in $\mu^{\mathrm{e}}$ also varying linearly with the effective mean normal stress [33]. Since neither $\varepsilon_{v}^{\mathrm{e}}$ nor $\varepsilon_{\mathrm{s}}^{\mathrm{e}}$ is zero at the post-gravity configuration, $\Psi$ takes on a nonzero value at this reference configuration.

Let us next consider a plasticity model based on modified Cam-Clay theory of critical state soil mechanics [32], reformulated in [33] to include finite deformation effects. In the small strain regime, the yield function takes the form

$$
\mathscr{F}=\frac{q^{2}}{M^{2}}+p\left(p-p_{c}\right)=0,
$$

where $p$ is the effective mean normal Cauchy stress, $q$ is the second invariant of the deviatoric component of the Cauchy stress tensor, $p_{c}$ is the preconsolidation pressure of the soil, and $M$ is a material parameter representing the slope of the critical state line on the $p-q$ space. In the finite strain regime, the yield function is obtained by replacing $p, q$ and $p_{c}$ in (4.3) by their Kirchhoff counterparts, $P=J p, Q=J q$, and $P_{c}$, respectively [33]. Central to the reformulated model is a bilogarithmic hardening law that is appropriate for cases involving large plastic volumetric strains [43]. The plasticity model described in [33] is coupled with the energy-conserving elasticity model described in the preceding paragraph through a formulation based on a multiplicative decomposition of the deformation gradient. This hyperelastic-plastic constitutive model is capable of replicating plastic volumetric compaction and dilation that are essential for testing the robustness of the nonlinear consolidation model.

The material parameters used in the one-dimensional simulations are shown in Table 1 . Two sets of compressibility parameters are used to describe the volume change behavior of the soil: $(\tilde{\kappa}, \tilde{\lambda})$ for the small strain case, and $(\hat{\kappa}, \hat{\lambda})$ for the large strain case. See [33] for a description of the physical significance of these parameters. The mass densities of the solid and fluid phases, $\rho_{\mathrm{s}}$ and $\rho_{\mathrm{w}}$, respectively, are assumed to be constant. These mass densities, together with the porosity $\varphi$ of the soil skeleton, may be used to determine the saturated mass density $\rho_{\mathrm{sar}}$ of the soil-water mixture through the expression

$$
\rho_{\text {sat }}=(1-\varphi) \rho_{\mathrm{s}}+\varphi \rho_{\mathrm{w}} .
$$

The same finite element mesh and boundary conditions shown in Fig. 1 are used in the present 1D simulation. The small strain initialization phase consists of applying the gravity loads, and then determining the internal stresses that balance these loads. Since the stored energy function (4.2) results in an elastic bulk modulus that is a linear function of the volumetric effective stress, nonzero initial stresses are required to get the solution

\begin{tabular}{|c|c|c|}
\hline Parameter & Small strain & Finite strain \\
\hline$\mu_{0}(\mathrm{kPa})$ & 200.0 & 200.0 \\
\hline$\alpha$ & 0.0 & 0.0 \\
\hline$\tilde{\kappa}$ & 0.0476 & - \\
\hline$\tilde{\lambda}$ & 0.1667 & - \\
\hline$\hat{\kappa}$ & - & 0.05 \\
\hline$\hat{\lambda}$ & - & 0.20 \\
\hline$M$ & 1.00 & 1.00 \\
\hline$p_{0}(\mathrm{kPa})$ & -10.0 & -10.0 \\
\hline$p_{c o}(\mathrm{kPa})$ & -10.0 & -10.0 \\
\hline$\varepsilon_{\mathrm{VO}}^{\mathrm{e}}$ & -0.0476 & -0.0500 \\
\hline$\rho_{\mathrm{s}}\left(\mathrm{t} / \mathrm{m}^{3}\right)$ & 2.70 & 2.70 \\
\hline$\rho_{w}\left(t / \mathrm{m}^{3}\right)$ & 1.00 & 1.00 \\
\hline$k$ (m/day) & $8.64 \times 10^{-4}$ & $8.64 \times 10^{-4}$ \\
\hline
\end{tabular}

Table 1

Material parameters for hyperelastic-plastic MCC model 

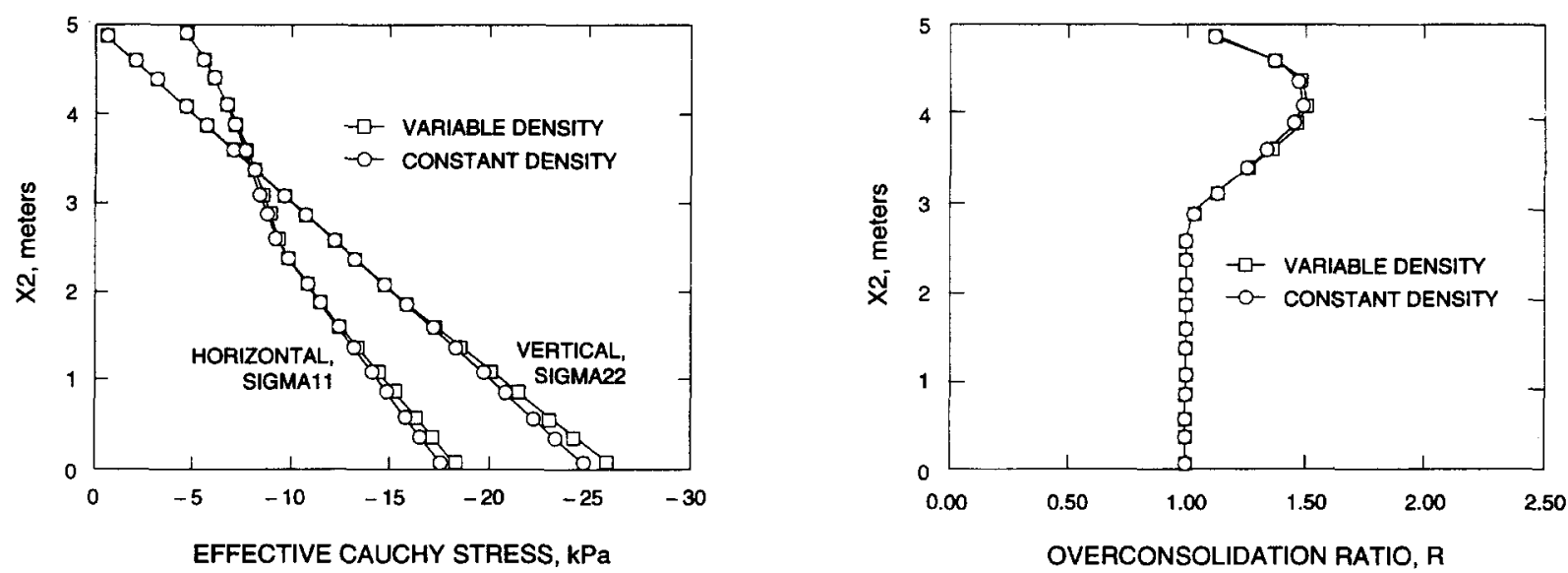

Fig. 4. One-dimensional hyperelastic-plastic consolidation: initial effective stresses generated by constant and variable weight density assumptions.

Fig. 5. One-dimensional hyperelastic-plastic consolidation: initial overconsolidation ratio $R$ generated by constant and variable weight density assumptions.

started. A small initial value of $-10.0 \mathrm{kPa}$ has been assumed at all Gauss points for the isotropic stress $p$ and the preconsolidation pressure $p_{c}$ for this initialization run.

Fig. 4 shows the horizontal $\left(\sigma_{11}\right)$ and vertical $\left(\sigma_{22}\right)$ Cauchy effective stress distributions generated by the gravity load. Together with the initial hydrostatic pore pressure distribution shown in Fig. 1, the stresses of Fig. 4 are the consistent internal stresses that balance this load. Two sets of solution are shown in Fig. 4 . The first solution shows the saturated mass density $\rho_{\text {sat }}$ varying with depth due to change in the value of the porosity $\varphi$ with depth as a result of increasing overburden load. This initial stress condition is useful for subsequent large-strain analysis. The second solution assumes a constant $\rho_{\text {sat }}=1.506 \mathrm{t} / \mathrm{m}^{3}$, and is useful for subsequent small-strain analysis. Since Fig. 4 shows that the initial conditions for the small and finite deformation analyses are nearly the same, a comparison of the results from these two analyses is meaningful.

Fig. 5 shows a plot of the variation of initial overconsolidation ratio of the soil with depth. For purposes of definition, the generalized overconsolidation ratio $R$ in a small strain setting is defined as

$$
R=\frac{p_{c}}{p}\left[1+\left(\frac{q}{M p}\right)^{2}\right]^{-1} \text {. }
$$

In a finite strain setting, the Kirchhoff stress quantities $P, Q$ and $P_{c}$ may be substituted in (4.5) in lieu of the Cauchy stress quantities $p, q$ and $p_{c}$. Thus, $R=1$ implies a normally consolidated soil $(\mathscr{F}=0)$, while $R>1$ implies an overconsolidated soil $(\mathscr{F}<0)$. Clearly, initially setting the effective isotropic stress $p$ to $-10 \mathrm{kPa}$ prior to the gravity load imposition phase causes the stress points near the ground surface to become overconsolidated due to insufficient overburden weight to make the stress points yield, while at greater depths the soil tends to become normally consolidated due to higher overburden loads. Fig. 5 shows that, again, the difference between the constant and variable mass density solutions is small, thus allowing for a meaningful comparison of the subsequent small and finite deformation analysis results.

A vertical downward load of $\Delta w^{\text {(Cauchy) }}=-90 \mathrm{kPa}$ was next applied at the top of the soil column in 3 time steps at a constant rate of $30.0 \mathrm{kPa} /$ day, with $\Delta t=1.0$ day each time step. Then, the resulting excess pore pressures were allowed to dissipate with time. The results employing small and large strain assumptions are shown in Fig. 6, which depicts a comparison of the variations with respect to time of the total fluid potential $I I$ at the same Gauss point $A$ shown in Fig. 1. Observe that the dissipation of hydraulic head is faster for the finite deformation solution due to a reduced drainage path resulting from the compaction of the soil column. Furthermore, the finite deformation solution exhibits a steady-state hydraulic potential of approximately $3.3 \mathrm{~m}$, which represents a $34 \%$ decrease in the total height of the soil column.

Fig. 7 shows a comparison of the isochrones of Cauchy pore pressures at different values of the equivalent time factor $T$ predicted by the small strain and finite deformation solutions. For purposes of definition, the 


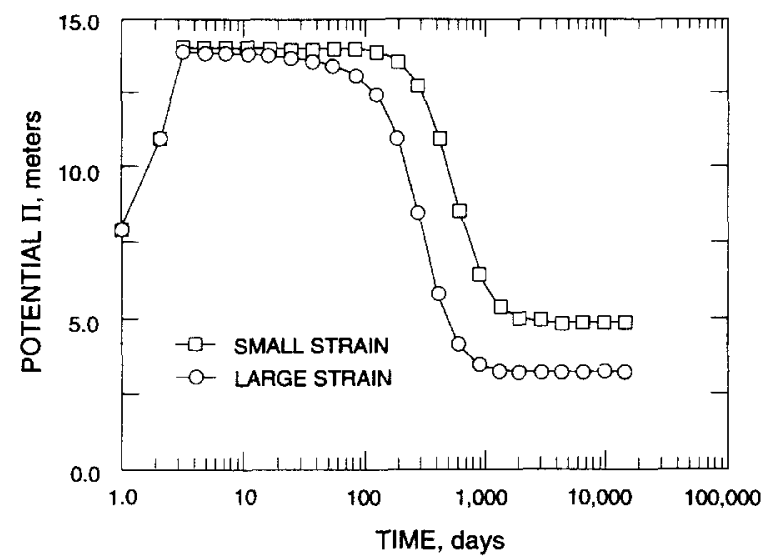

Fig. 6. One-dimensional hyperelastic-plastic consolidation: variation of total potential at Gauss point $A$ with time.

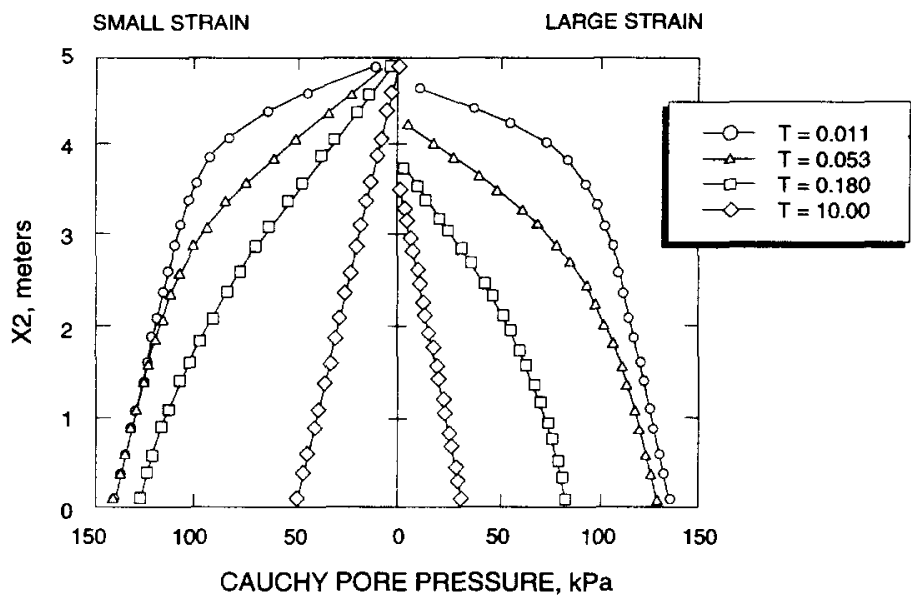

Fig. 7. One-dimensional hyperelastic-plastic consolidation: isochrones of constant Cauchy pore pressures.

equivalent time factor $T$ for the hyperelastic-plastic case is calculated as in Section 4.1 as $T=c_{\mathrm{v}} t / H_{0}^{2}$, where $c_{\mathrm{v}}=2 \mu_{0} k_{\mathrm{v}} /\left(\rho_{\mathrm{w}} \mathrm{g}\right)$ and $k_{\mathrm{v}}=k$ in Table 1 . Again, the isochrones predicted by the latter solution reflect a moving domain resulting from the inclusion of finite deformation effects. A comparison of the average degrees of consolidation $\bar{U}_{\text {ave }}$ predicted by the two solutions is depicted in Fig. 8. Here, $\bar{U}_{\text {ave }}$ is defined as the ratio between the time-varying ground surface settlement to the ultimate settlement at the end of consolidation. Note that the small strain solution predicts a slower rate of consolidation than the finite deformation solution because the latter solution considers explicitly the reduction in length of the drainage path, which enhances the dissipation of excess pore pressures. (This effect could be offset by a reduction of the coefficient of permeability of the soil as it consolidates, but this factor has not been taken into consideration in the present analysis.) Geometric effects are usually considered negligible in routine calculations, but this example shows that they can change the character of the solution when the deformation is large.

\subsection{Plane strain hyperelastic consolidation}

Closed-form solutions are available for the problem of plane strain consolidation of an elastic half-space subjected to a uniform strip load [44]. In this example, we will attempt to replicate these solutions numerically and demonstrate the significance of finite deformation effects on the response of a consolidating hyperelastic soil medium deforming in plane strain.

Fig. 9 shows the finite element mesh used for the two-dimensional plane-strain problem. The problem consists of an embankment load of half-width $a=5 \mathrm{~m}$ applied over a hyperelastic soil layer $20 \mathrm{~m}$ thick. The mesh is 


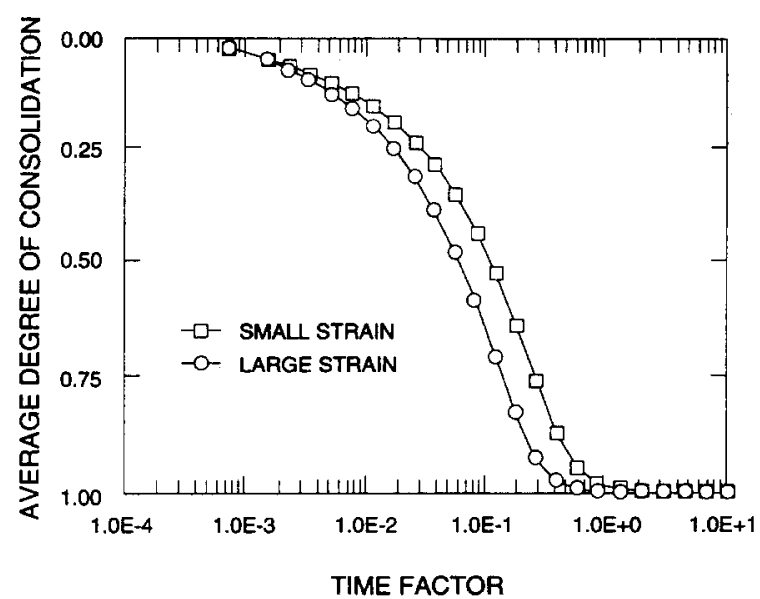

Fig. 8. One-dimensional hyperelastic-plastic consolidation: average degree of consolidation versus time factor.
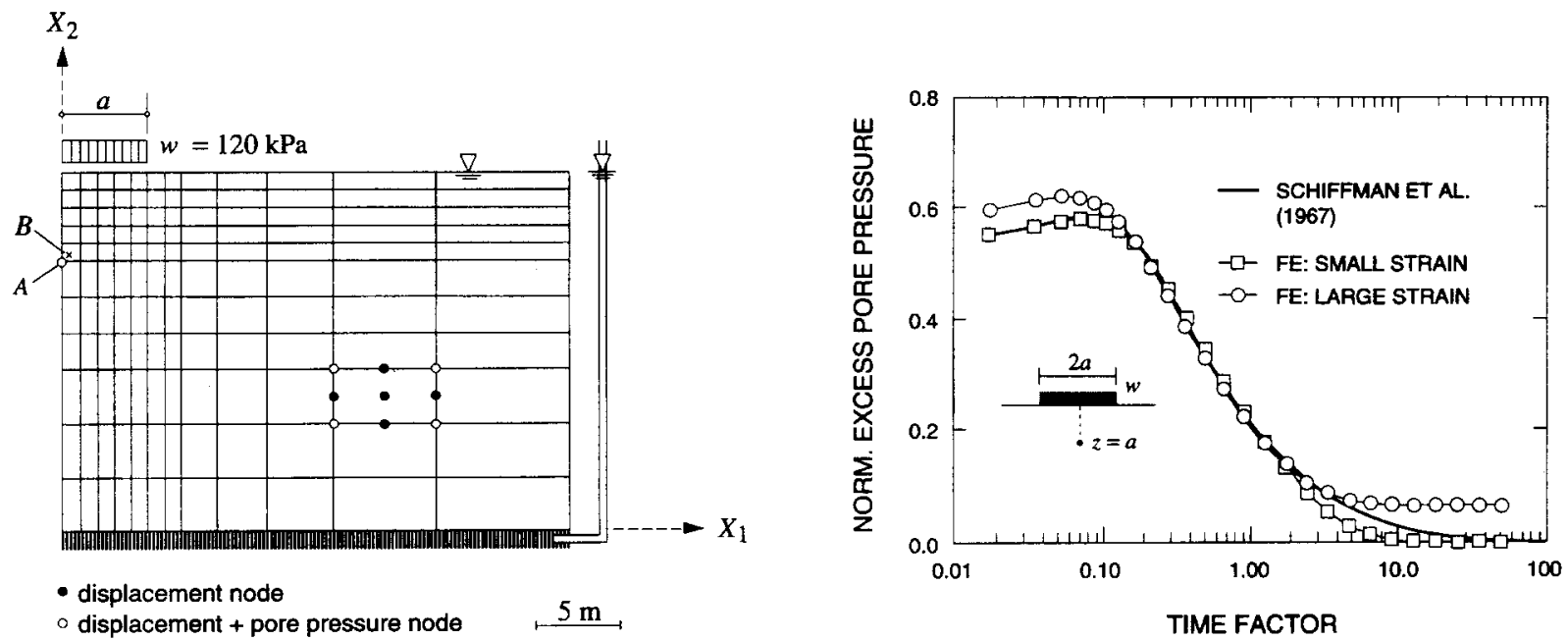

Fig. 9. FE mesh for plane strain hyperelastic consolidation example.

Fig. 10. Plane strain hyperelastic consolidation: variation of centerline excess pore pressure at depth $z=a$ with time.

composed of 132 D9P4 mixed elements with 575 displacement nodes and 156 pore pressure nodes. The bottom of the clay layer is assumed to be rigid, rough, and perfectly draining, and subjected to a constant value of total potential equal to $\Pi=20.0 \mathrm{~m}$. The material parameters are $\lambda=0$ and $\mu=250 \mathrm{kPa}$ (corresponding to Young's modulus $E=500 \mathrm{kPa}$ and Poisson's ratio $\nu=0$ ). A strip load of $w^{(\text {Cauchy })}=120 \mathrm{kPa}$ is applied nearly instantaneously (over a period of $\Delta t=10$ days, which is very small compared to $t \approx 10000$ days required to reach complete consolidation) at the ground surface, and is then held constant while the soil undergoes consolidation. Permeabilities are $k=k_{11}=k_{22}=8.64 \times 10^{-4} \mathrm{~m} /$ day, and $k_{12}=k_{21}=0$; fluid mass density is $\rho_{\mathrm{w}}=1.0 \mathrm{ton} / \mathrm{m}^{3}$. The soil elements are assumed to be initially stress-free.

Fig. 10 shows the closed-form solution for the time-variation of the centerline excess pore pressure at depth $z=a$ beneath the strip load on a semi-infinite elastic halfspace [44]. Along with this solution are the predictions of the numerical model. For convenience, the excess pore pressures have been normalized with respect to the embankment load intensity $w$ according to the expression $\left(\vartheta-\vartheta_{0}\right) / w$, where $\vartheta_{0}$ is the reference hydrostatic Cauchy pore pressure. The point corresponding to $z=a$ in the mesh of Fig. 9 is node $A$, which is situated at a depth of $5 \mathrm{~m}$ from the base of the embankment. The small strain FE solution readily provides the time variation of the pore pressure at this point, since node $A$ is a pore pressure node. However, the large strain model needs the values of the Jacobian to determine the Cauchy pore pressures, which are not readily available at the nodal 
points. Thus, we choose the nearest Gauss point to node $A$ to assess the accuracy of the numerical model. For consistency in presentation, both the small-strain and large-strain FE solutions are evaluated at Gauss point $B$ located at horizontal and vertical distances of $0.211 \mathrm{~m}$ from node $A$, see Fig. 9. A normalized time factor, $T=c t / a^{2}$, where $c=2 \mu k /\left(\mathrm{g} \rho_{\mathrm{w}}\right)$ and $t$ is elapsed time since the beginning of the consolidation, is used to describe the solutions in the time domain.

A comparison of the curves shown in Fig. 10 suggests that higher pore pressures are induced in the large strain case by the sudden application of the external load at the early stage of the consolidation process. Thereafter, the dissipation occurs at almost the same rate up to a time factor $T \approx 5.0$, when the large strain solution stabilizes while the small strain solution is still decreasing. Note that the large strain solution asymptotically approaches a nonzero excess pore pressure since the final steady-state pore pressure is numerically different from the initial hydrostatic pore pressure due to the variation in the geometric configuration of the problem. As expected, the small strain FE solution agrees better with the closed-form solution, but is not identical to it because of the limitation of the FE model in representing a halfspace and because of the use of a finite time increment to impose the embankment load, among other factors. Both the closed-form and FE solutions exhibit the Mandel-Cryer effect, or the initial increase in excess pore pressure, which is a characteristic feature of the coupled solution [40].

Fig. 11 shows the isochrones of constant Cauchy pore pressures predicted by the small and finite deformation models along the vertical line $X_{1}=0.211 \mathrm{~m}$ beneath the embankment load. This line is defined by the column of Gauss points closest to the axis of symmetry of the problem (i.e. the centerline). Note that the large strain solution predicts a steady-state isochrone defined by a nearly straight line with an apparent slope equivalent to a fluid with mass density of about 1.23 tons $/ \mathrm{m}^{3}$, which is greater than the assumed fluid mass density of $\rho_{\mathrm{w}}=1.0 \mathrm{ton} / \mathrm{m}^{3}$. This is a result of a local artesian condition characterized by steady-state upward seepage created by the reduction in thickness of the consolidating layer, as the top and bottom drainage boundary conditions remain unchanged. The Cauchy pore pressure at the bottom boundary converges toward a steady-state value that is slightly higher than the initial value. This is a consequence of prescribing the essential boundary condition in the form of Kirchhoff pore pressure, which was amplified by the inverse of the Jacobian that is less than unity due to volumetric compression of the soil.

\subsection{Plane strain hyperelastic-plastic consolidation}

As a final example, we consider the problem of a strip flexible footing resting on a compressible clay that has been subjected previously to a surface preload. Preloading creates an initially overconsolidated state that makes the response of the soil stiffer upon subsequent reloading. The FE mesh used for this problem is shown in Fig. 12. Here, the clay deposit is represented by the same mesh of Fig. 9, but a $1 \mathrm{~m}$-thick layer of sand elements represented by 12 D9P0 finite elements is now placed on top of the clay layer to simulate a drainage blanket.

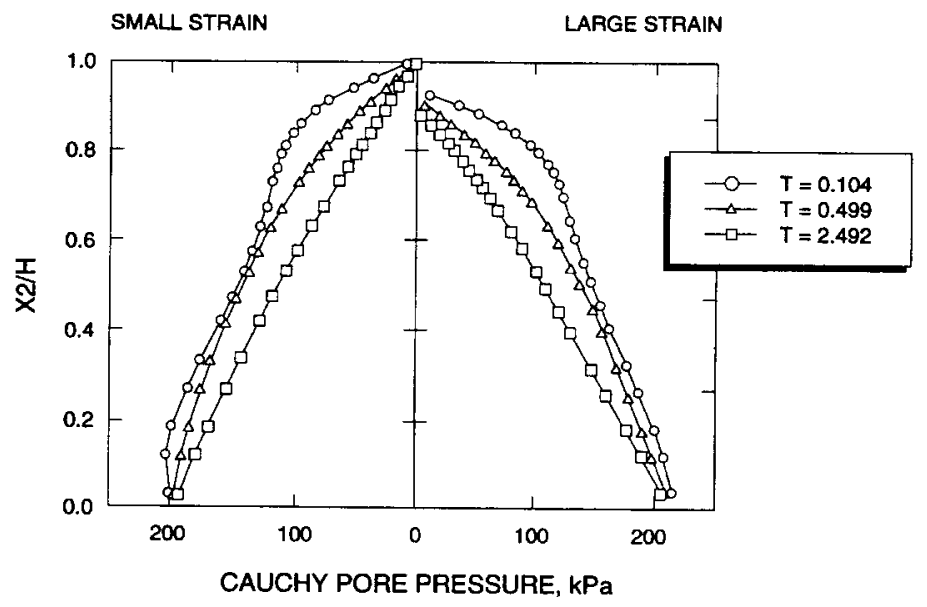

Fig. 11. Plane strain hyperelastic consolidation: isochrones of constant Cauchy pore pressures along vertical line $\mathrm{XI}=0.211 \mathrm{~m}$ near centerline. 


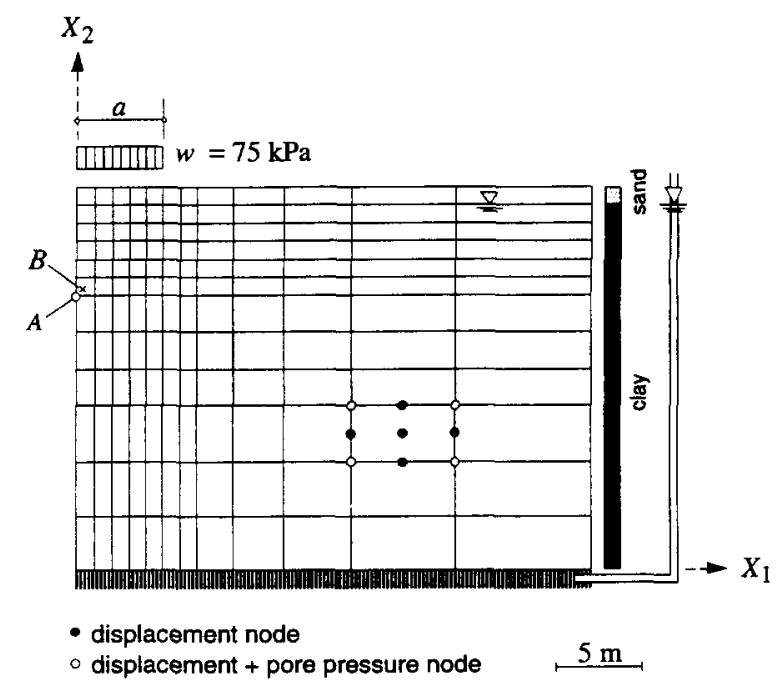

Fig. 12. FE mesh for plane strain hyperelastic-plastic consolidation example.

The top sand layer is modeled as a hyperelastic material with material parameters shown in Table 2 . A reference porosity $\varphi_{0}$ is required by the large strain model since it calculates the macroscopic mass density of the soil as a function of porosity. The underlying soft clay is modeled as a hyperelastic-plastic modified Cam-Clay material with the same material parameters shown in Table 1.

As in the example of Section 4.2, the initialization procedure entails running small strain analyses to generate the initial reference configurations produced by the gravity loads, with and without consideration of the variation of porosity with depth. The soil is next preloaded to surface loads of $30 \mathrm{kPa}, 15 \mathrm{kPa}$, and zero (normally consolidated), producing three states of soil overconsolidation. For the case of $30 \mathrm{kPa}$-preload, Figs. 13 and 14 show the variations with depth of the initial state of stress and overconsolidation ratio $R$ of the soil. The results of Figs. 13 and 14 show the horizontal effective stresses varying nonlinearly with depth due to 'locked-in' stresses produced by the preload, but are otherwise nearly the same for the two runs, as are the vertical effective stresses and the overconsolidation ratio profiles. Similar results are obtained for the zero- and $15 \mathrm{kPa}$-preload cases. The initial conditions generated above are next utilized to study the small and large strain consolidation responses of the foundation soil to a surface embankment load.

Next, a strip load of intensity $w=75 \mathrm{kPa}$ was applied over a half-width of $a=5 \mathrm{~m}$ at a constant rate over a period of 90 days, after which it was held constant. Again, this amount of time is very small in comparison to the time required to reach complete consolidation, and so the soil essentially behaves in an undrained fashion during the embankment load application stage. Fig. 15 shows the evolution of the ground surface centerline settlements with depth as predicted by the large strain analyses, along with the prediction of the small strain analysis for the extreme case of no preload. As expected, the large strain analyses predict smaller values of vertical displacements. These displacements in turn decrease with increasing values of the overconsolidation ratio $R$.

Fig. 16 shows the evolution of the Cauchy pore pressure $\vartheta=\theta / J$ at Gauss point $B$ (see Fig. 12). Note that the large strain solution predicts lower initial excess pore pressures but slightly higher final steady-state pore

Table 2

Material parameters for sand layer

\begin{tabular}{lll}
\hline Parameter & Small strain & Finite strain \\
\hline$E(\mathrm{kPa})$ & 1000.0 & 1000.0 \\
$\nu$ & 0.00 & 0.00 \\
$\varphi_{0}$ & - & 0.41 \\
$\rho_{\mathrm{s}}\left(\mathrm{t} / \mathrm{m}^{3}\right)$ & - & 2.70 \\
$\rho_{\mathrm{w}}\left(\mathrm{t} / \mathrm{m}^{3}\right)$ & - & 1.00 \\
$\rho\left(\mathrm{t} / \mathrm{m}^{3}\right)$ & 2.01 & - \\
\hline
\end{tabular}



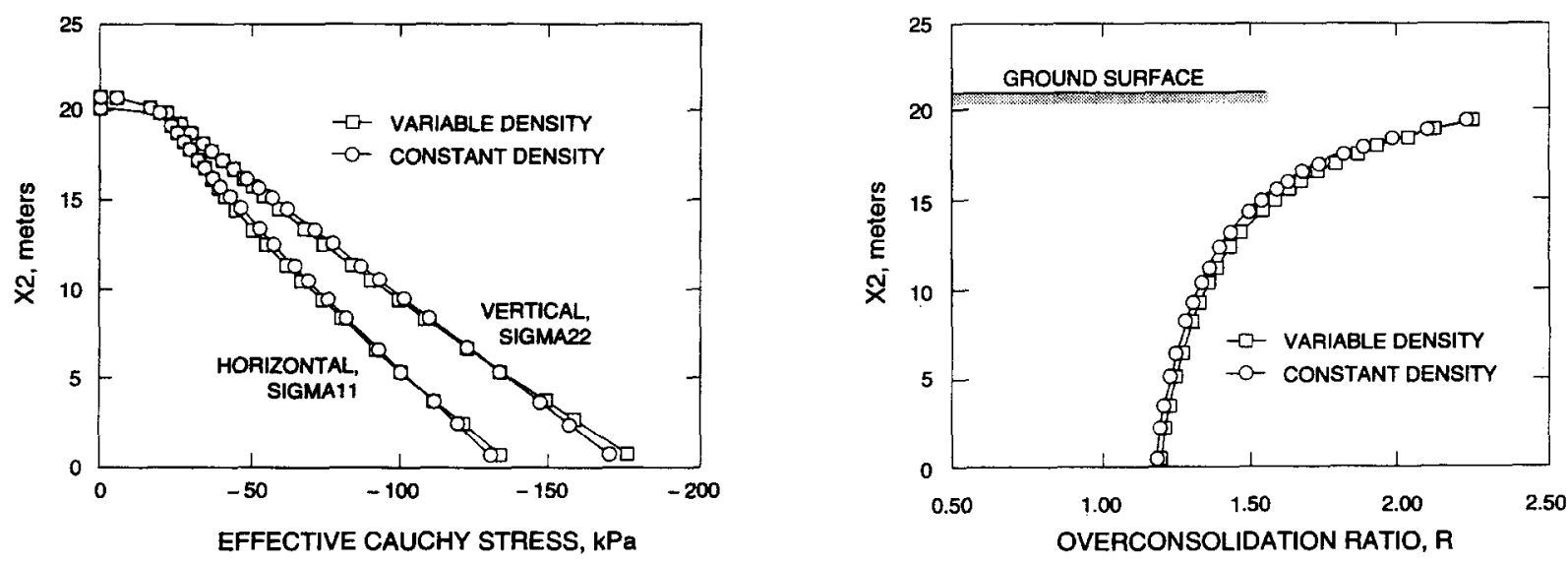

Fig. 13. Plane strain hyperelastic-plastic consolidation: initial effective stresses generated by constant and variable weight density assumptions.

Fig. 14. Plane strain hyperelastic-plastic consolidation: initial overconsolidation ratio $R$ generated by constant and variable weight density assumptions.

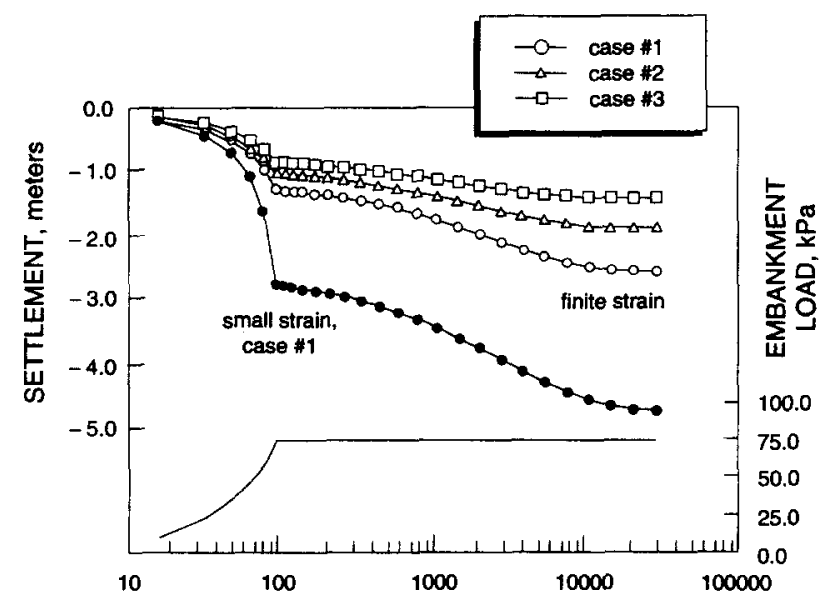

Fig. 15. Plane strain hyperelastic-plastic consolidation: ground surface settlement at centerline versus time (case \#1: no preload; case \#2: $15 \mathrm{kPa}$ preload; case \#3: $30 \mathrm{kPa}$ preload).

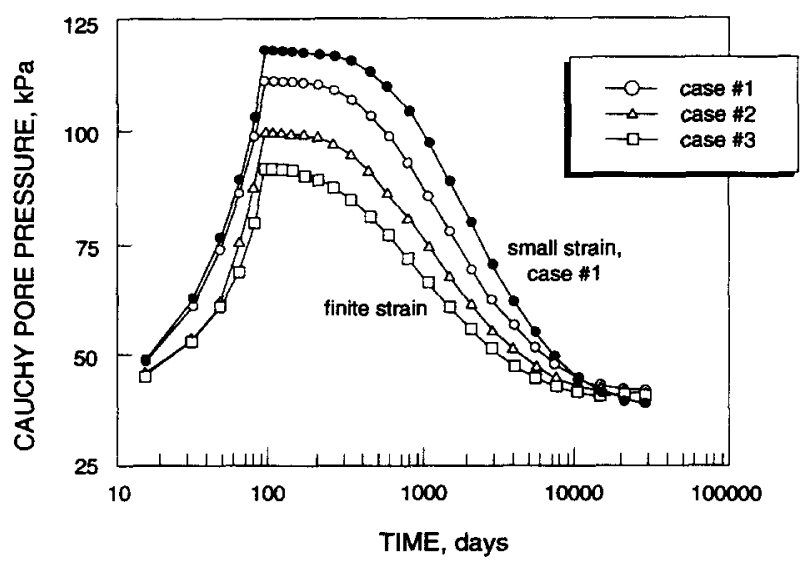

Fig. 16. Plane strain hyperelastic-plastic consolidation: Cauchy pore pressure at point $B$ versus time (case \#1: no preload; case \#2: $15 \mathrm{kPa}$ preload; case \#3: $40 \mathrm{kPa}$ preload). 
pressures. The final steady-state pore pressures predicted by the large-strain model reflect the same local artesian condition created by considering the finite reduction in thickness of the consolidating layer similar to the effect described in the example of Section 4.3.

Fig. 17 compares the deformed meshes at time instants $t=90,1482$ and 29349 days predicted by the large deformation and small strain models for the case in which the soil was preloaded to $15 \mathrm{kPa}$. Superimposed in these figures are the zones of plastification (shaded region) characterized by the condition $R=1$. The rigid wedge effect is evident from the deformed meshes as thin prisms of overconsolidated clay persist to form directly beneath the embankment load. However, observe that the small strain solution predicts a more widespread zone of plastification than does the finite deformation solution.

The results of Fig. 17 are elaborated further in Figs. 18 and 19, which show a comparison of the effective Cauchy and Kirchhoff stress paths at the same Gauss point $B$ predicted by the small strain and finite deformation models, respectively (recall that the finite deformation constitutive model is formulated in terms of the Kirchhoff stresses). Note that during the embankment loading stage the undrained effective stress paths predicted by the small-strain model have essentially reached the critical state line, implying that the soil is already at or near failure at the end of the embankment loading stage. On the other hand, for the same loading the finite deformation model still predicts subfailure conditions in all three cases. Consequently, the small strain model could not produce convergent results when the embankment load exceeded $75 \mathrm{kPa}$. In contrast, the finite deformation model could accommodate embankment loads as high as $90 \mathrm{kPa}$. As for the undrained effective stress paths in the overconsolidated range, vertical slopes are predicted by both models, implying uncoupled volumetric and deviatoric elastic responses associated with the use of the hyperelastic equation (4.2), with $\alpha=0$ $[33,34,42]$.

Figs. 18 and 19 show that during consolidation the stress points move away from the critical state line, causing the yield surface to expand further. Consolidation creates a condition in which the Jacobian $J$ continually decreases with time as a result of the volumetric compaction of the soil skeleton (in contrast, the value of $J$ is equal to unity during undrained loading, and hence, the values of the Cauchy and Kirchhoff stresses remain identical during this period). Consequently, the soil experiences a gain of shear strength due to strain hardening. A comparison of the expanded yield loci depicted in the two figures suggests that the small

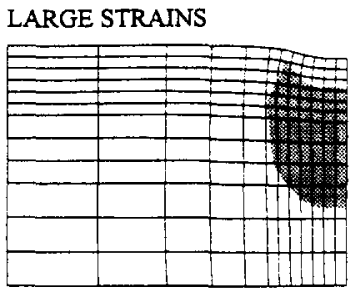

STEP \#6: $t=90$ days

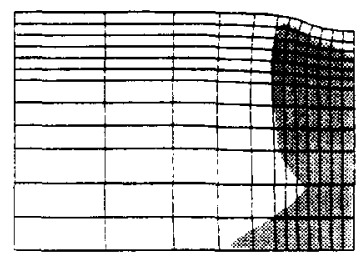

STEP \#18: $t=1482$ days

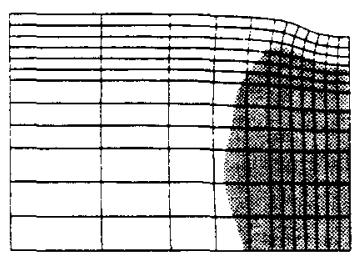

STEP \#27: $t=29349$ days
SMALL STRANS
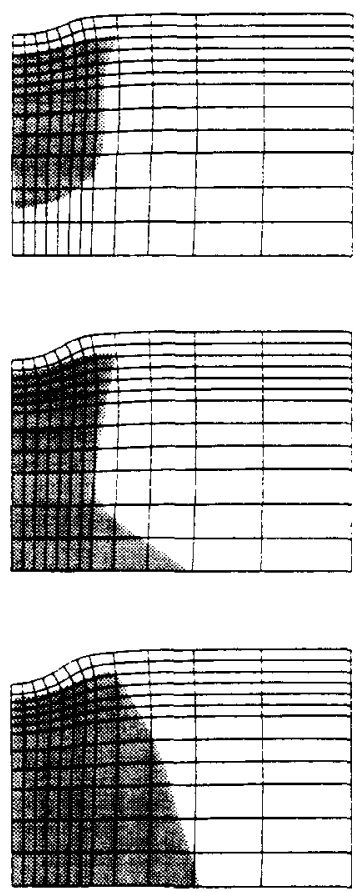

Fig. 17. Deformed meshes and zones of plastification for plane strain hyperelastic-plastic consolidation examples. 

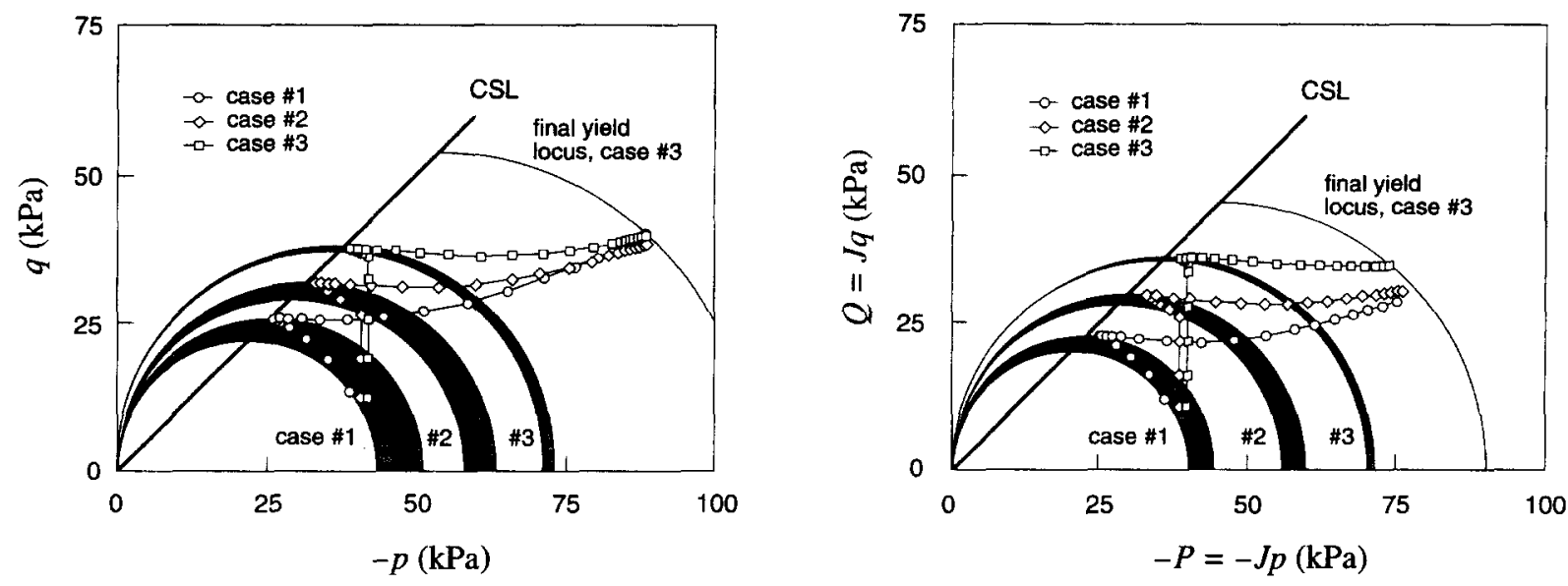

Fig. 18. Small strain analysis: effective Cauchy stress paths for Gauss point $B$ showing expansion of yield loci during undrained loading (case \#1: no preload; case \#2: $15 \mathrm{kPa}$ preload; case \#3: $30 \mathrm{kPa}$ preload).

Fig. 19. Large strain analysis: effective Kirchhoff stress paths for Gauss point $B$ showing expansion of yield loci during undrained loading (case \#1: no preload; case \#2: $15 \mathrm{kPa}$ preload; case \#3: $30 \mathrm{kPa}$ preload).

strain model predicts more intense plastification than does the finite deformation model during both the undrained loading and the consolidation stages.

Finally, Table 3 compares typical convergence profiles exhibited by Newton-Raphson iterations during the undrained embankment loading and consolidation stages. The analyses pertain to the worst-case scenario of no preload (initially normally consolidated soil profile), where the solution requires the most number of iterations to converge. Note that the undrained loading stage is driven in the initial iteration by the unbalanced residual forces $\boldsymbol{r}_{\phi}$ of (3.12) arising from the incremental embankment load, whereas the consolidation stage is driven by the unbalanced fluid flow $\boldsymbol{r}_{\theta}$ of (3.13) due to diffusion effects (which in turn is converted into unbalanced forces $\boldsymbol{r}_{\phi}$ during the next iteration, causing the relative residual norm at iteration $\# 1$ of the consolidation analysis, $\left\|\boldsymbol{r}^{\mathrm{I}}\right\| /\left\|\boldsymbol{r}^{\mathrm{O}}\right\|$, to jump up about two orders of magnitude, see Table 3). Interestingly, the large strain solution typically converges one iteration faster than the small strain solution during the embankment loading stage, but the trend is reversed during the consolidation stage. All in all, this example shows that consistent linearization

Table 3

Typical quadratic convergence of Newton-Raphson iterations: iteration number versus normalized norm of residual

\begin{tabular}{lcc}
\hline Iteration number & Small strain & Finite strain \\
\hline (a) Time step \#6: Undrained loading, $t=90$ & days \\
0 & $1.000 \mathrm{e}+0$ & $1.000 \mathrm{e}+0$ \\
1 & $8.398 \mathrm{e}-1$ & $8.689 \mathrm{e}-1$ \\
2 & $6.359 \mathrm{e}-1$ & $2.714 \mathrm{e}-1$ \\
3 & $2.927 \mathrm{e}-1$ & $4.461 \mathrm{e}-2$ \\
4 & $7.720 \mathrm{e}-2$ & $4.238 \mathrm{e}-4$ \\
5 & $4.903 \mathrm{e}-3$ & $5.530 \mathrm{e}-8$ \\
6 & $2.301 \mathrm{e}-5$ & $2.127 \mathrm{e}-12$ \\
7 & $5.530 \mathrm{e}-10$ & - \\
& & \\
(b) Time step \#18: Consolidation, $t=1482$ days \\
0 & $1.000 \mathrm{e}+0$ & $1.000 \mathrm{e}+0$ \\
1 & $1.075 \mathrm{e}+2$ & $1.085 \mathrm{e}+2$ \\
2 & $2.342 \mathrm{e}+1$ & $2.132 \mathrm{e}+1$ \\
3 & $7.175 \mathrm{e}-1$ & $1.576 \mathrm{e}+0$ \\
4 & $3.450 \mathrm{e}-3$ & $1.189 \mathrm{e}-3$ \\
5 & $7.092 \mathrm{e}-8$ & $2.123 \mathrm{e}-6$ \\
6 & - & $1.048 \mathrm{e}-8$ \\
\hline
\end{tabular}


makes running a finite deformation elastoplastic consolidation analysis just as manageable as running an equivalent nonlinear small strain analysis.

\section{Summary and conclusions}

A mathematical model based on multiplicative plasticity for finite strain elasto-plastic consolidation of fully saturated soil media has been implemented into a finite element program. The solid phase is described by a hyperelastic-plastic version of modified Cam-Clay model capable of representing plastic volumetric compaction that accompanies the compression of clayey soils. Fluid flow is described by the generalized Darcy's law formulated with respect to the current configuration. The two constitutive laws are both amenable to consistent linearization in closed form.

Numerical examples involving one-dimensional compression and two-dimensional plane strain loading on compressible clays demonstrate the usefulness of the finite deformation model. Specifically, a plane-strain example problem was run on clays that have varying degrees of overconsolidation to show that, unlike the commonly used hypoelastic-based finite strain models which are restricted to small elastic strains, the new formulation can also accommodate for the development of large elastic strains. In addition, the formulation used in the model circumvents the rate issue in finite deformation analysis. A comparison of results of small strain and finite deformation analyses show that large deformation effects can significantly influence the predicted deformation and pore pressure responses of the soil, as well as the time-variation of the average degree of consolidation. Results further indicate that consistent linearization makes running a finite deformation elastoplastic consolidation analysis just as manageable as running an equivalent nonlinear small strain analysis.

\section{Acknowledgments}

Funding for this research was provided in part by the Air Force Office of Scientific Research under Grant Number F49620-92-J-0008, and by the G3S Division of National Science Foundation under Contract No. MSS-9022448. Financial support was likewise provided to the first author by the Ministerio de Educación y Ciencia de España while Part 1 of this paper was being written in Madrid. Professors G. Calabresi (University of Rome, La Sapienza) and J.K. Mitchell (Virginia Polytechnic Institute) provided the much appreciated motivation and inspirational support.

\section{References}

[1] L. Bjerrum, Engineering geology of Norwegian normally consolidated marine clays as related to settlements of buildings, 7th Rankine Lecture, Géotechnique 17 (1967) 83-117.

[2] K. Terzaghi, Theoretical Soil Mechanics (Wiley, New York, 1943).

[3] M.A. Biot, General theory of three-dimensional consolidation, J. Appl. Phys. 12 (1941) 155-164

[4] M.A. Biot, Theory of elasticity and consolidation for a porous anisotropic solid, J. Appl. Phys. 26 (1955) 182-185.

[5] M.A. Biot, Theory of propagation of elastic waves in a fluid-saturated porous solid, .J. Acoust. Soc. Am. 28 (1956) $168-191$.

[6] M.A. Biot, Mechanics of deformation and acoustic propagation in porous media, J. Appl. Phys. 33 (1967) 1483-1498.

[7] R. de Boer, R.L. Schiffman and R.E. Gibson, The origins of the theory of consolidation: The Terzaghi-Fillunger dispute, Géotechnique 46 (1996) $175-186$.

[8] R.J. Atkin and R.E. Craine, Continuum theories of mixtures: basic theory and historical development, Q.J. Mech. Appl. Math. 29 (1976) 209-244.

[9] R.M. Bowen, Theory of mixtures, in: A.C. Eringen, ed., Continuum Physics 3 (Academic Press, New York, 1976) 1-27.

[10] A.C. Eringen and J.D. Ingram, A continuum theory of chemically reacting media-I, Int. J. Engrg. Sci. 3 (1965) $197-212$.

[11] J.D. Ingram and A.C. Eringen, A continuum theory of chemically reacting media-II: Constitutive equations of reacting fluid mixtures, Int. J. Engrg. Sci. 5 (1967) 289-322.

[12] J.R. Booker and J.C. Small, An investigation of the stability of numerical solutions of Biot's equations of consolidation, Int. J. Solids Struct. 11 (1975) 907-971.

[13] J.T. Christian, Two- and three-dimensional consolidation, in: C.S. Desai and J.T. Christian, eds., Numerical Methods in Geotechnical Engineering (McGraw-Hill, San Francisco, 1977) 399-426.

[14] R.L. Schiffman, The stress components of a porous media, J. Geophys. Res, 75 (1970) 4035-4038. 
[15] R.I. Borja, Nonlinear consolidation: linear multistep methods and iterative algorithms, in: G. Beer, J.R. Booker and J.P. Carter, eds., Proc. Seventh Int. Conf. Comput. Methods Advances Geomech. (Balkema, 1991) 1111-1116.

[16] R.I. Borja, One-step and linear multistep methods for nonlinear consolidation, Comput. Methods Appl. Mech. Engrg. 85 (1991) 239-272.

[17] R.I. Borja, Composite Newton-PCG and quasi-Newton iterations for nonlinear consolidation, Comput. Methods Appl. Mech. Engrg. 88 (1991) 27-60.

[18] A.M. Britto and M.J. Gunn, Critical State Soil Mechanics via Finite Elements (John Wiley and Sons, New York, 1987).

[19] H.J. Siriwardane and C.S. Desai, Two numerical schemes for nonlinear consolidation, Int. J. Numer. Methods Engrg. 17 (1981) $405-426$.

[20] J.C. Small, J.R. Booker and E.H. Davis, Elasto-plastic consolidation of soils, Int. J. Solids Struct. 12 (1976) 431-448.

[21] J.H. Prevost, Mechanics of continuous porous media, Int. J. Engrg. Sci. 18 (1980) 787-800.

[22] J.H. Prevost, Implicit-explicit schemes for nonlinear consolidation, Comput. Methods Appl. Mech. Engrg. 39 (1983) $225-239$.

[23] J.P. Carter, J.R. Booker and J.C. Small, The analysis of finite elasto-plastic consolidation, Int. J. Numer. Analyt. Methods Geomech. 3 (1979) $107-129$.

[24] A.E. Green and P.M. Naghdi, A general theory of an elastic-plastic continuum, Arch. Rat. Mech. Anal. 18 (1965) 251-281.

[25] A.E. Green and B.C. McInnis, Generalized hypo-elasticity, Proc. Roy. Soc. Edinburgh A57 (1967) 220-230.

[26] T.J.R. Hughes, Numerical implementation of constitutive models: Rate independent deviatoric plasticity, in: S. Nemat-Nasser, R. Asaro and G. Hegemier, eds., Theoretical Foundations for Large-Scale Computations of Nonlinear Material Behaviour (Martinus Nijhoff, The Netherlands, 1984).

[27] J.C. Simo, Algorithms for static and dynamic multiplicative plasticity that preserve the classical return mapping schemes of the infinitesimal theory, Comput. Methods Appl. Mech. Engrg. 99 (1992) 61-112.

[28] R.I. Borja and E. Alarcón, A mathematical framework for finite strain elasto-plastic consolidation. Part 1: Balance laws, variational formulation, and linearization, Comput. Methods Appl. Mech. Engrg. 122 (1995) 145-171.

[29] J.C. Nagtegaal and J.E. de Jong, Some aspects of non-isotropic work-hardening in finite strain plasticity, in: E.H. Lee and R.L. Mallet, eds., Plasticity of Metals at Finite Strains, Proc. Research Workshop, Stanford University (1981) 65-102.

[30] R.I. Borja, Elasto-plastic consolidation at finite strain, in: H.J. Siriwardane and M.M. Zaman, eds., Computer Methods and Advances in Geomechanics 1 (A.A. Balkema, 1994) 753-758.

[31] A. Gens and D.M. Potts, Critical state models in computational geomechanics, Engrg. Comput. 5 (1988) 178-197.

[32] K.H. Roscoe and J.B. Burland, On the generalized stress-strain behaviour of 'wet' clay, in: J. Heyman and F.A. Leckie, eds., Engineering Plasticity (Cambridge University Press, 1968) 535-609.

[33] R.I. Borja and C. Tamagnini, Cam-Clay plasticity. Part III. Extension of the infinitesimal model to include finite strains, Comput. Methods Appl. Mech. Engrg., 155 (1998) 73-95.

[34] R.I. Borja, C. Tamagnini and A. Amorosi, Coupling plasticity and energy-conserving elasticity models for clays, J. Geotech. Geoenviron. Engrg. 123 (1997) 948-957.

[35] R.I. Borja and C. Tamagnini, Finite deformation theory for a Cam-Clay model, in: G.N. Pande and S. Pietruszczak, eds., Numerical Models in Geomechanics, NUMOG V (1995) 3-8.

[36] R.I. Borja and C. Tamagnini, Critical state model at finite strains, in: Y.K. Lin and T.C. Su, eds., Proc. 11th ASCE Engrg. Mech. Conf. 1 (1996) $148-151$

[37] T.J.R. Hughes, The Finite Element Method (Prentice-Hall, Englewood Cliffs, NJ, 1987).

[38] T.J.R. Hughes and J.H. Prevost, DIRT II-A Nonlinear Quasi-Static Finite Element Analysis Program (User's Manual. Stanford University, 1979).

[39] J.E. Marsden and T.J.R. Hughes, Mathematical Foundations of Elasticity (Prentice-Hall, Englewood Cliffs, NJ, 1983).

[40] T.W. Lambe and R.V. Whitman, Soil Mechanics (Wiley, New York, 1969).

[41] J. Bear, Dynamics of Fluids in Porous Media (Dover, New York, 1972).

[42] G.T. Houlsby, The use of a variable shear modulus in elastic-plastic models for clays, Comput. Geotech. 1 (1985) 3-13.

[43] R. Butterfield, A natural compression law for soils, Géotechnique 29 (1979) 469-480.

[44] R.L. Schiffman, A. Chen and J.C. Jordan, The Consolidation of a Half Plane, MATE Report 67-3 (U. Illinois, Chicago Circle, 1967). 